\title{
Strain-Based Analysis for Geometrically Nonlinear Beams: A Modal Approach
}

\author{
Weihua Su* \\ University of Alabama, Tuscaloosa, Alabama 35487-0280 \\ and \\ Carlos E. S. Cesnik \\ University of Michigan, Ann Arbor, Michigan 48109-2140 \\ DOI: $10.2514 / 1 . C 032477$
}

\begin{abstract}
A strain-based geometrically nonlinear finite element beam formulation for structural dynamic and aeroelastic analysis of slender beams and wings has been successfully applied to study the coupled nonlinear aeroelasticity and flight dynamics of different, very flexible aircraft. To improve the ability to use such aeroelastic framework in control design, a modal-based approach is developed in this paper to solve the strain-based nonlinear beam equations with a reduced number of degrees of freedom. The modal approach is applied to study geometrically nonlinear static and transient problems of constrained and free slender beams, subject to external structural or aerodynamic excitations. Accuracy and performance of the new formulation are compared with the direct nonlinear finite element procedure.
\end{abstract}

\begin{tabular}{|c|c|c|}
\hline \multicolumn{3}{|r|}{ Nomenclature } \\
\hline$A$ & $=$ & amplitude of oscillation \\
\hline$a_{0}$ & $=$ & $\begin{array}{l}\text { local aerodynamic frame, with } a_{0 y} \text { axis aligned } \\
\text { with zero lift line of airfoil }\end{array}$ \\
\hline$a_{1}$ & $=$ & $\begin{array}{l}\text { local aerodynamic frame, with } a_{1 y} \text { axis aligned } \\
\text { with airfoil motion velocity }\end{array}$ \\
\hline$B$ & $=$ & body reference frame \\
\hline$B^{F}, B^{M}$ & $=$ & $\begin{array}{l}\text { influence matrices for distributed forces and } \\
\text { moments }\end{array}$ \\
\hline$b$ & $=$ & $\begin{array}{l}\text { positions and orientations of } B \text { frame, as time } \\
\text { integral of } \beta\end{array}$ \\
\hline$b_{c}$ & $=$ & semichord of airfoil, $\mathrm{m}$ \\
\hline $\begin{array}{r}C_{F F}, C_{F B} \\
C_{B F}, C_{B B}\end{array}$ & $=$ & components of generalized damping matrix \\
\hline $\begin{array}{l}\bar{C}_{F F}, \bar{C}_{F B} \\
\bar{C}_{B F}, \bar{C}_{B B}\end{array}$ & $=$ & $\begin{array}{l}\text { components of generalized damping matrix in } \\
\text { modal equations }\end{array}$ \\
\hline$C^{B a_{1}}$ & $=$ & rotation matrix from $a_{1}$ frame to $B$ frame \\
\hline$C^{G B}$ & $=$ & rotation matrix from $B$ frame to $G$ frame \\
\hline$c$ & $=$ & chord length, m \\
\hline$d$ & $=$ & $\begin{array}{l}\text { distance of midchord in front of beam reference } \\
\text { axis, } m\end{array}$ \\
\hline$F_{1}, F_{2}, F_{3}$ & $=$ & $\begin{array}{l}\text { influence matrices in inflow equations with } \\
\text { independent variables }\end{array}$ \\
\hline $\bar{F}_{1 F}, \bar{F}_{2 F}$ & $=$ & $\begin{array}{l}\text { flexible components of influence matrices } F_{1} \\
\text { and } F_{2} \text { in the modal equations }\end{array}$ \\
\hline$F^{\text {aero }}, M^{\text {aero }}$ & $=$ & nodal aerodynamic forces and moments \\
\hline$F^{\mathrm{dist}}, F^{\mathrm{pt}}$ & $=$ & distributed and point forces \\
\hline$G$ & $=$ & global (inertial) reference frame \\
\hline & $=$ & gravity acceleration column matrix, $\mathrm{m} / \mathrm{s}^{2}$ \\
\hline$H_{h b}$ & & $\begin{array}{l}\text { matrix consisting of influence from Jacobian } J_{h b} \\
\text { and body angular velocities } \omega_{B}\end{array}$ \\
\hline$h$ & $=$ & absolute positions and rotations of beam nodes \\
\hline
\end{tabular}

Presented as Paper 2012-1713 at the 53rd AIAA/ASME/ASCE/AHS/ASC Structures, Structural Dynamics, and Materials Conference, Honolulu, HI, 23-26 April 2012; received 14 June 2013; revision received 19 September 2013; accepted for publication 29 September 2013; published online 11 March 2014. Copyright () 2013 by Weihua Su and Carlos E. S. Cesnik. Published by the American Institute of Aeronautics and Astronautics, Inc., with permission. Copies of this paper may be made for personal or internal use, on condition that the copier pay the $\$ 10.00$ per-copy fee to the Copyright Clearance Center, Inc., 222 Rosewood Drive, Danvers, MA 01923; include the code 1542-3868/14 and \$10.00 in correspondence with the CCC.

*Assistant Professor, Department of Aerospace Engineering and Mechanics; suw@eng.ua.edu. Senior Member AIAA.

†Professor, Department of Aerospace Engineering; cesnik@umich.edu. Fellow AIAA.

\begin{tabular}{|c|c|c|}
\hline$J$ & $=$ & Jacobian matrix \\
\hline$\underline{K}_{F F}$ & $=$ & components of the generalized stiffness matrix \\
\hline $\bar{K}_{F F}$ & $=$ & $\begin{array}{l}\text { components of the generalized stiffness matrix in } \\
\text { modal equations }\end{array}$ \\
\hline$L$ & $=$ & beam length or wingspan, $m$ \\
\hline$l_{\mathrm{mc}}$ & $=$ & aerodynamic loads on an airfoil about its \\
\hline$m_{\mathrm{mc}}, d_{\mathrm{mc}}$ & & midchord \\
\hline$l_{\mathrm{ra}}, m_{\mathrm{ra}}, d_{\mathrm{ra}}$ & $=$ & $\begin{array}{l}\text { aerodynamic loads on an airfoil about its beam } \\
\text { reference axis }\end{array}$ \\
\hline$M, C, K$ & $=$ & $\begin{array}{l}\text { discrete mass, damping, and stiffness matrices of } \\
\text { whole system }\end{array}$ \\
\hline $\begin{array}{r}M_{F F}, M_{F B}, \\
M_{B F}, M_{B B}\end{array}$ & $=$ & components of generalized mass matrix \\
\hline $\begin{array}{r}\bar{M}_{F F}, \bar{M}_{F B} \\
\bar{M}_{B F}, \bar{M}_{B B}\end{array}$ & $=$ & $\begin{array}{l}\text { components of generalized mass matrix in modal } \\
\text { equations }\end{array}$ \\
\hline$M^{\mathrm{dist}}, M^{\mathrm{pt}}$ & $=$ & distributed and point moments \\
\hline$m$ & $=$ & mass per span, $\mathrm{kg} / \mathrm{m}$ \\
\hline$N$ & $=$ & influence matrix for gravity force \\
\hline$P_{B}$ & $=$ & inertia position of $B$ frame, resolved in $G$ frame \\
\hline$p_{B}, \theta_{B}$ & $=$ & $\begin{array}{l}\text { position and orientation of } B \text { frame, as time } \\
\text { integral of } v_{B} \text { and } \omega_{B} \text {, respectively }\end{array}$ \\
\hline & $=$ & position of $w$ frame with respect to $B$ frame \\
\hline$R_{B}, R_{F}$ & $=$ & $\begin{array}{l}\text { rigid-body and flexible components of general- } \\
\text { ized load vector }\end{array}$ \\
\hline $\bar{R}_{B}, \bar{R}_{F}$ & $=$ & $\begin{array}{l}\text { rigid-body and flexible components of general- } \\
\text { ized load vector in modal equations }\end{array}$ \\
\hline$s$ & $=$ & beam curvilinear coordinate, $\mathrm{m}$ \\
\hline$t$ & $=$ & time, s \\
\hline$W^{\text {ext }}, W^{\text {int }}$ & $=$ & external and internal virtual works, respectively \\
\hline$w$ & $=$ & $\begin{array}{l}\text { local beam reference frame defined at each node } \\
\text { along beam reference line }\end{array}$ \\
\hline$\dot{y}, \dot{z}$ & $=$ & $\begin{array}{l}\text { airfoil translational velocity components re- } \\
\text { solved in } a_{0} \text { frame, } \mathrm{m} / \mathrm{s}\end{array}$ \\
\hline$\dot{\alpha}$ & $=$ & airfoil angular velocity about $a_{0 x}$ axis, $\mathrm{rad} / \mathrm{s}$ \\
\hline$\beta$ & $=$ & $\begin{array}{l}\text { body velocities, with translational and angular } \\
\text { components, resolved in } B \text { frame }\end{array}$ \\
\hline$\varepsilon$ & $=$ & $\begin{array}{l}\text { total elastic strain variable of aircraft, as a } \\
\text { column matrix }\end{array}$ \\
\hline & $=$ & element elastic strain variable \\
\hline$\varepsilon^{0}$ & $=$ & initial (prescribed) elastic strain value \\
\hline$\zeta$ & $=$ & quaternions defining orientation of $B$ frame \\
\hline$\eta$ & $=$ & modal coordinates \\
\hline$\theta$ & $=$ & rotations of beam nodes, rad \\
\hline$\lambda$ & $=$ & inflow states, $\mathrm{m} / \mathrm{s}$ \\
\hline$\lambda_{0}$ & $=$ & inflow velocities, $\mathrm{m} / \mathrm{s}$ \\
\hline$v_{B}, \omega_{B}$ & $=$ & $\begin{array}{l}\text { linear and angular velocities of } B \text { frame, resolved } \\
\text { in } B \text { frame itself }\end{array}$ \\
\hline
\end{tabular}




$\begin{array}{ll}\rho & =\text { air density, } \mathrm{kg} / \mathrm{m}^{3} \\ \Phi & \text { (strain) normal mode shape } \\ \Phi_{C}, \Phi_{F}, \Phi_{B}= & \text { complete, flexible (strain), and rigid-body com- } \\ & \text { ponents of the strain-based mode shape, } \\ & \text { respectively } \\ \Omega_{\zeta}(\beta) & \begin{array}{l}\text { coefficient matrix of quaternion equations, a } \\ \text { function of body angular velocities }\end{array}\end{array}$

Subscripts

$B$

$B B, B F=$ components of a matrix with respect to body/ flexible differential equations of motion

$F \quad=\quad$ reference to flexible degrees of freedom

$F B, F F=$ components of a matrix with respect to flexible/ body differential equations of motion

$h b=h$ with respect to motion of $B$ frame

$h \varepsilon \quad=h$ with respect to strain $\varepsilon$

$p b \quad=\quad$ nodal position with respect to motion of $B$ frame

$p \varepsilon \quad=$ nodal position with respect to strain $\varepsilon$

$\theta b=$ nodal rotation with respect to motion of $B$ frame

$\theta \varepsilon \quad=\quad$ nodal rotation with respect to strain $\varepsilon$

\section{Introduction}

$\mathbf{I}_{\mathrm{a}}^{\mathrm{N}}$ N STUDIES of very flexible aircraft (e.g., gliders and highaltitude long-endurance aircraft), the slender wings of these vehicles can be modeled as beams by taking advantage of their structure slenderness. However, the high flexibility associated with them brings some special requirements to the beam formulation applied to the analysis. From previous investigations [1], the slender wings of very flexible aircraft may undergo large motions, although still under small strain, under normal operating loads, exhibiting geometrically nonlinear behavior. The structural dynamic and aeroelastic characteristics of the aircraft may change significantly due to large deflections of their flexible wings. In addition, very flexible aircraft usually see coupling between the low-frequency elastic modes of their slender wings and the rigid-body motions of the complete aircraft [1-5]. Therefore, the coupled effects between the large deflection due to the wing flexibility and the aeroelastic/flight dynamic characteristics of the complete aircraft must be properly accounted for in a nonlinear aeroelastic solution. For this purpose, a beam formulation that is able to capture the geometrically nonlinear wing deformation is required to serve as the basis of the nonlinear structural dynamic and aeroelastic analysis of very flexible aircraft.

There have been different geometrically nonlinear formulations established for slender beams. One may classify the formulations based on the solution methodology, namely, the displacement-based formulation (e.g., [6]), the mixed-form formulation (e.g., [7-9]), and the strain-based (or stress-based) formulation (e.g., [10,11]). Their differences lie in the definition of independent variables to represent the displacement field and the treatment of the beam reference line's rotation in the solution. A brief description of the three types of beam formulations was provided by $\mathrm{Su}$ and Cesnik [12], whereas a comprehensive discussion on the three types of formulations for the structural, aeroelastic, and flight dynamic analysis of very flexible aircraft was done by Palacios et al. [13]. They compared the solutions in terms of their numerical efficiency and simplicity for integrated flight dynamic analysis with full aircraft flexibility.

A complete treatment of the strain-based geometrically nonlinear beam formulation was introduced by Su and Cesnik [12]. The strainbased formulation defines the extensional strain, bending, and twist curvatures of the beam reference line as the independent degrees of freedom, whereas transverse shears are not explicitly included in them. Unlike some strain-based formulations presented in the literature (e.g., [10]), which enforce the beam equilibrium equation and strain-displacement kinematics simultaneously with Lagrange multipliers, the formulation introduced in [12] solves these equations iteratively. Thus, the derived governing equation is the classic form of a second-order differential equation, which simplifies the solution process. For that, the force and displacement boundary conditions are considered in a different way than in [10]. These improvements in this formulation make it more flexible in modeling arbitrary beam configurations under different loading conditions. Besides the aforementioned advantages, the strain-based formulation brings additional benefits to control studies since the curvatures are the variables that can be directly measured by the strain sensors. This formulation may also demonstrate great computational efficiency due to the reduction in degrees of freedom for the same complex deformation when compared to the displacement-based or mixedform formulations. Lastly, the strain-based formulation is efficient in solving geometrically nonlinear static problems, as it features a constant stiffness matrix (for statically determinate beams). This advantage, however, does not hold in nonlinear transient solutions, where the inertia and damping of the system need to be updated according to the instantaneous beam deformation states.

Different solution techniques have been applied to solve the developed geometrically nonlinear beam formulation. A common approach is to use the well-developed finite element method. Numerous applications of the finite element method can be found in the literature. The strainbased beam formulation introduced in [12] was also solved by using this approach. Generally, finite element solutions may converge to the exact solution as the mesh is refined. However, the dimension of the problem may dramatically increase with the refinement of the mesh, which brings higher computational cost for the solution. Since a fast solution is always desired in the nonlinear structural dynamic and aeroservoelastic analysis of very flexible aircraft, other solution techniques have also been developed and applied in studies.

In addition to the finite element method, the modal approach is another popular technique that is widely used in engineering analysis. One of its important advantages is its significant reduction in the dimension of the problem. Moreover, normal modes in the modal analysis may decouple the system matrices and further simplify the problem. One may also find significant characteristics of the vibrational behaviors by using modal approaches. Because of the advantages of modal approaches, they have been applied in various studies to solve the beam dynamics and aeroelasticity of very flexible wings and aircraft. Tang and Dowell [14-16] performed both experimental and numerical studies on the nonlinear flutter boundary, gust response, and limit-cycle oscillations of a slender cantilever beam. In their analytical study, general mode shape functions were applied to solve the nonlinear beam equations in terms of generalized coordinates. Patil and Althoff [17] presented a Galerkin approach for the solution of the nonlinear beam equations. In this study, the mixedform intrinsic geometrically nonlinear beam equations of motion including the kinematic equations and boundary conditions [9] were weighted by appropriate function to represent the rate of change of internal energies and the work done by external forces. In their numeric analysis about a slender beam, they demonstrated the advantage of the Galerkin method over the finite element method in terms of the convergence speed and accuracy. Palacios [18] applied nonlinear normal modes method to solve the mixed-form intrinsic nonlinear beam equations. The nonlinear modal equations in intrinsic coordinates were obtained from integrals involving only products of the mode shapes and known sectional properties. The nonlinear normal modes were sought through an asymptotic approximation to the invariant manifolds that defines them in the space of intrinsic modal coordinates. Dynamics of isotropic and anisotropic beams were solved with this method.

The strain-based nonlinear beam formulation [12] has been employed by the Shearer and Cesnik [3], Su and Cesnik [4,5], Cesnik and Brown [19], and Cesnik and Sü [20] for solving aeroelastic problems. Common in these applications is that the beam formulation was solved in a finite element approach. In this paper, a modal solution of the strain-based beam formulation is introduced, which can potentially facilitate future control studies, where a low number of degrees of freedom is highly desirable. As the beam dynamics derived in [12] are represented by the strain (curvature) and its time derivatives, the normal modes used in this paper are strain modes. The numerical study demonstrates the accuracy and reduction of the problem size in the modal solution approach. However, because of the nature of the strain-based beam formulation, there is a tradeoff 
between those advantages and the computational cost of the solution when using the modal approach.

\section{Theoretical Formulation}

The finite element solution of the strain-based geometrically nonlinear beam formulation has been discussed by Su and Cesnik [12]. An introduction of the derivation of the finite element equations of motion with the modal transformation is presented here for completeness.

\section{A. System Frames}

As shown in Fig. 1a, a fixed global (inertial) frame $G$ is first defined. A body frame $B(t)$ is then built in the global frame to describe the vehicle position and orientation, with $B_{x}(t)$ pointing to the right wing, $B_{y}(t)$ pointing forward, and $B_{z}(t)$ being the cross product of $B_{x}(t)$ and $B_{x}(t)$. The position and orientation $b$ and the time derivatives, $\dot{b}$ and $b$, of the $B$ frame can be defined as

$$
\begin{aligned}
& b=\left\{\begin{array}{c}
p_{B} \\
\theta_{B}
\end{array}\right\} \quad \dot{b}=\beta=\left\{\begin{array}{c}
\dot{p}_{B} \\
\dot{\theta}_{B}
\end{array}\right\}=\left\{\begin{array}{c}
v_{B} \\
\omega_{B}
\end{array}\right\} \\
& \ddot{b}=\dot{\beta}=\left\{\begin{array}{l}
\ddot{p}_{B} \\
\ddot{\theta}_{B}
\end{array}\right\}=\left\{\begin{array}{c}
\dot{v}_{B} \\
\dot{\omega}_{B}
\end{array}\right\}
\end{aligned}
$$

where $p_{B}$ and $\theta_{B}$ are body position and orientation, both resolved in the body frame $B$. Note that the origin of the body frame is arbitrary in the vehicle, and it does not have to be the location of the vehicle's center of gravity.

Within the body frame, a local beam frame $w$ is built at each node along the beam reference line (Fig. 1b), which is used to define the nodal position and orientation. Vectors $\boldsymbol{w}_{x}(s, t), \boldsymbol{w}_{y}(s, t)$, and $\boldsymbol{w}_{z}(s, t)$ are bases of the beam frame for which the directions are pointing along the beam reference axis, toward the leading edge (LE) or the front direction, and normal to the beam surface, respectively, resolved in the body frame. The curvilinear beam coordinate is $s$.

\section{B. Elements with Constant Strains}

In [12], a nonlinear beam element was introduced to model the elastic deformation of slender beams. Strain degrees (curvatures) of the beam reference line were considered as the independent variables in the solution. The strain-based formulation allows simple shape functions for the element. Constant-value functions are used here. Thus, the strain variable of an element is denoted as

$$
\varepsilon_{e}^{T}=\left\{\begin{array}{llll}
\varepsilon_{x} & \kappa_{x} & \kappa_{y} & \kappa_{z}
\end{array}\right\}
$$

where $\varepsilon_{x}$ is the extensional strain; and $\kappa_{x}, \kappa_{y}$, and $\kappa_{z}$ are the twist of the beam reference line, bending about the local $w_{y}$ axis and the local $w_{z}$ axis, respectively. The total strain variable of the complete aircraft is obtained by assembling the elemental strains:

$$
\varepsilon^{T}=\left\{\begin{array}{llll}
\varepsilon_{e 1}^{T} & \varepsilon_{e 2}^{T} & \varepsilon_{e 3}^{T} & \ldots
\end{array}\right\}
$$

Transverse shear strains are not explicitly included in this equation. However, shear strain effects are included in the constitutive relation [21]. Complex geometrically nonlinear deformations can be represented by such a constant strain distribution over each element. To represent the quadratic nodal displacement field that will be recovered from the constant strain, the element is defined with three equally spaced nodes. Some of the functions, such as inertia and distributed load, are assumed to vary linearly between the three nodes of each element. The values of these functions over the element can be obtained from its nodal values using linear Lagrange interpolation functions.

\section{Nodal Displacement and Jacobians}

The position and orientation of each node along the beam are defined by a column matrix consisting of 12 components, denoted as

$$
h(s)^{T}=\left\{\left[p_{B}+p_{w}(s)\right]^{T} \quad w_{x}(s)^{T} \quad w_{y}(s)^{T} \quad w_{z}(s)^{T}\right\}
$$

where $p_{w}$ is the position of the $w$ frame resolved in the body frame. The derivatives and variations of the dependent variable $h$ are related with those of the independent ones as

$\begin{array}{ll}\delta h=J_{h \varepsilon} \delta \varepsilon+J_{h b} \delta b & \dot{h}=J_{h \varepsilon} \dot{\varepsilon}+J_{h b} \dot{b}=J_{h \varepsilon} \dot{\varepsilon}+J_{h b} \beta \\ d h=J_{h \varepsilon} d \varepsilon+J_{h b} d b & \ddot{h}=J_{h \varepsilon} \ddot{\varepsilon}+\dot{J}_{h \varepsilon} \dot{\varepsilon}+J_{h b} \dot{\beta}+\dot{J}_{h b} \beta\end{array}$

where

$$
J_{h \varepsilon} \equiv \frac{\partial h}{\partial \varepsilon} \quad J_{h b} \equiv \frac{\partial h}{\partial b}
$$

are the transformation Jacobians obtained from the beam kinematics. The other necessary Jacobian matrices, including $J_{p \varepsilon}, J_{\theta \varepsilon}, J_{p b}$, and $J_{\theta b}$, which relate the nodal positions and rotations to the independent variables, can be derived from $J_{h \varepsilon}$ and $J_{h b}[\underline{3}, 12]$.

\section{Finite Element Equations of Motion}

The equations of motion of the system are derived by following the principle of virtual work extended to dynamic systems (equivalent to Hamilton's principle). The total virtual work done on a beam is found by integrating the products of all internal and external forces and the corresponding virtual displacements over the volume, which is given as

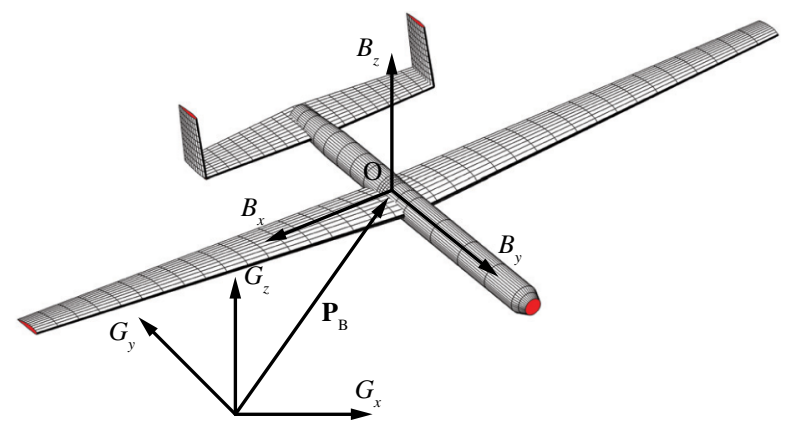

a) Global and body frame defining the rigid-body motion of aircraft

Fig. 1 Basic beam reference frames. 


$$
\delta W=\int_{V} \delta u^{T}(x, y, z) f(x, y, z) \mathrm{d} V
$$

where $f$ represents general forces acting on a differential volume. This may include internal elastic forces, inertial forces, gravity forces, external distributed forces and moments, external point forces and moments, etc. The corresponding virtual displacement is $\delta u$. When beam cross-sectional properties are known, the integration of Eq. (7) over the beam volume is simplified to the integration along the beam coordinate $s$. This integration is first numerically performed over each beam element, followed by the assemblage of the element quantities to obtain the total virtual work on the whole beam. This paper gives a brief overview of the equations of motion, and the detailed derivation process can be found in $[4,12]$. The virtual work due to internal and external loads is given by

$$
\begin{aligned}
& \delta W^{\mathrm{int}}=-\delta h^{T} M \ddot{h}-\delta h^{T} M H_{h b} \beta-\delta h^{T} M \dot{J}_{h b} \beta-\delta \varepsilon^{T} C \dot{\varepsilon}-\delta \varepsilon^{T} K\left(\varepsilon-\varepsilon^{0}\right) \\
& \delta W^{\mathrm{ext}}=-\delta h^{T} N g+\delta p_{w}^{T} B^{F} F^{\mathrm{dist}}+\delta \theta^{T} B^{M} M^{\mathrm{dist}}+\delta p_{w}^{T} F^{\mathrm{pt}}+\delta \theta^{T} M^{\mathrm{pt}}
\end{aligned}
$$

The dependent variables $\left(h, p_{w}\right.$, and $\left.\theta\right)$ can be replaced by the independent variable by applying the Jacobians [see Eq. (6)] and their subsets. Therefore, the total virtual work on a beam can be written as

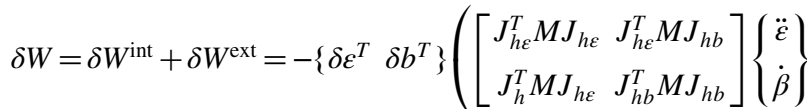

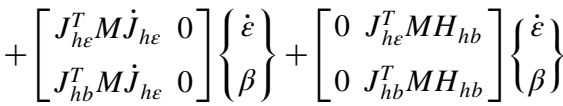

$$
\begin{aligned}
& \left.+\left[\begin{array}{cc}
0 & 2 J_{h \varepsilon}^{T} M \dot{J}_{h b} \\
0 & 2 J_{h b}^{T} M \dot{J}_{h b}
\end{array}\right]\left\{\begin{array}{l}
\dot{\varepsilon} \\
\beta
\end{array}\right\}+\left[\begin{array}{cc}
C & 0 \\
0 & 0
\end{array}\right]\left\{\begin{array}{l}
\dot{\varepsilon} \\
\beta
\end{array}\right\}+\left[\begin{array}{cc}
K & 0 \\
0 & 0
\end{array}\right]\left\{\begin{array}{l}
\varepsilon \\
b
\end{array}\right\}-\left\{\begin{array}{c}
K \varepsilon^{0} \\
0
\end{array}\right\}\right)
\end{aligned}
$$

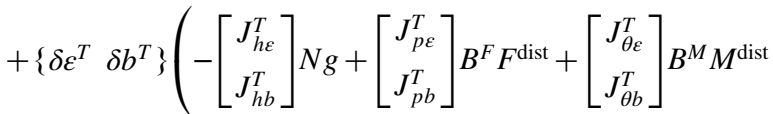

$$
\begin{aligned}
& \left.+\left[\begin{array}{c}
J_{p \varepsilon}^{T} \\
J_{p b}^{T}
\end{array}\right] F^{\mathrm{pt}}+\left[\begin{array}{c}
J_{\theta \varepsilon}^{T} \\
J_{\theta b}^{T}
\end{array}\right] M^{\mathrm{pt}}\right)
\end{aligned}
$$

Following the same process described in [4] , the elastic equations of motion are eventually derived as

$$
\begin{gathered}
{\left[\begin{array}{ll}
M_{F F} & M_{F B} \\
M_{B F} & M_{B B}
\end{array}\right]\left\{\begin{array}{l}
\ddot{\varepsilon} \\
\dot{\beta}
\end{array}\right\}+\left[\begin{array}{ll}
C_{F F} & C_{F B} \\
C_{B F} & C_{B B}
\end{array}\right]\left\{\begin{array}{l}
\dot{\varepsilon} \\
\beta
\end{array}\right\}} \\
+\left[\begin{array}{cc}
K_{F F} & 0 \\
0 & 0
\end{array}\right]\left\{\begin{array}{l}
\varepsilon \\
b
\end{array}\right\}=\left\{\begin{array}{l}
R_{F} \\
R_{B}
\end{array}\right\}
\end{gathered}
$$

where the generalized inertia, damping, and stiffness matrices are

$$
\begin{aligned}
& M_{F F}(\varepsilon)=J_{h \varepsilon}^{T} M J_{h \varepsilon} \quad M_{F B}(\varepsilon)=J_{h \varepsilon}^{T} M J_{h b} \\
& M_{B F}(\varepsilon)=J_{h b}^{T} M J_{h \varepsilon} \quad M_{B B}(\varepsilon)=J_{h b}^{T} M J_{h b} \\
& C_{F F}(\varepsilon, \dot{\varepsilon}, \beta)=C+J_{h \varepsilon}^{T} M \dot{J}_{h \varepsilon} \quad C_{F B}(\varepsilon, \dot{\varepsilon}, \beta)=J_{h \varepsilon}^{T} M H_{h b}+2 J_{h \varepsilon}^{T} M \dot{J}_{h b} \\
& C_{B F}(\varepsilon, \dot{\varepsilon}, \beta)=J_{h b}^{T} M \dot{J}_{h \varepsilon} \quad C_{B B}(\varepsilon, \dot{\varepsilon}, \beta)=J_{h b}^{T} M H_{h b}+2 J_{h b}^{T} M \dot{J}_{h b} \\
& K_{F F}=K
\end{aligned}
$$

and the generalized force vector is

$$
\begin{gathered}
\left\{\begin{array}{c}
R_{F} \\
R_{B}
\end{array}\right\}=\left\{\begin{array}{c}
K_{F F} \varepsilon^{0} \\
0
\end{array}\right\}-\left[\begin{array}{c}
J_{h \varepsilon}^{T} \\
J_{h b}^{T}
\end{array}\right] N g+\left[\begin{array}{c}
J_{p \varepsilon}^{T} \\
J_{p b}^{T}
\end{array}\right] B^{F} F^{\mathrm{dist}} \\
+\left[\begin{array}{c}
J_{\theta \varepsilon}^{T} \\
J_{\theta b}^{T}
\end{array}\right] B^{M} M^{\mathrm{dist}}+\left[\begin{array}{c}
J_{p \varepsilon}^{T} \\
J_{p b}^{T}
\end{array}\right] F^{\mathrm{pt}}+\left[\begin{array}{c}
J_{\theta \varepsilon}^{T} \\
J_{\theta b}^{T}
\end{array}\right] M^{\mathrm{pt}}
\end{gathered}
$$

$N, B^{F}$, and $B^{M}$ are the influence matrices for the gravity force, distributed forces, and distributed moments, respectively, which come from the numerical integration. The generalized force vector involves the effects from initial strains $\varepsilon^{0}$, gravity fields $g$, distributed forces $F^{\text {dist }}$, distributed moments $M^{\text {dist }}$, point forces $F^{\text {pt }}$, and point moments $M^{\mathrm{pt}}$. The aerodynamic forces and moments are considered as distributed loads.

\section{E. Unsteady Aerodynamics}

The distributed loads $F^{\text {dist }}$ and $M^{\text {dist }}$ in Eq. (12) are divided into aerodynamic loads and user-supplied loads. The unsteady aerodynamic loads used in the current study are based on the two dimensional (2-D) finite-state inflow theory provided in [22]. The theory calculates aerodynamic loads on a thin airfoil section undergoing large motions in an incompressible inviscid subsonic flow. The lift, moment, and drag of a thin 2-D airfoil section about its midchord are given by

$$
\begin{aligned}
& l_{\mathrm{mc}}=\pi \rho_{\infty} b_{c}^{2}(-\ddot{z}+\dot{y} \dot{\alpha}-d \ddot{\alpha})+2 \pi \rho_{\infty} b_{c} \dot{y}^{2}\left[-\frac{\dot{z}}{\dot{y}}+\left(\frac{1}{2} b_{c}-d\right) \frac{\dot{\alpha}}{\dot{y}}-\frac{\lambda_{0}}{\dot{y}}\right] \\
& m_{\mathrm{mc}}=\pi \rho_{\infty} b_{c}^{2}\left(-\frac{1}{8} b_{c}^{2} \ddot{\alpha}-\dot{y} \dot{z}-d \dot{y} \dot{\alpha}-\dot{y} \lambda_{0}\right) \\
& d_{\mathrm{mc}}=-2 \pi \rho_{\infty} b_{c}\left(\dot{z}^{2}+d^{2} \dot{\alpha}^{2}+\lambda_{0}^{2}+2 d \dot{z} \dot{\alpha}+2 \dot{z} \lambda_{0}+2 d \dot{\alpha} \lambda_{0}\right)
\end{aligned}
$$

where $b_{c}$ is the semichord, and $d$ is the distance of the midchord in front of the reference axis. The quantity $-\dot{z} / \dot{y}$ is the angle of attack that consists of the contributions from both the pitching angle and the unsteady plunging motion of the airfoil. The different velocity components are shown in Fig. 2.

The inflow parameter $\lambda_{0}$ accounts for induced flow due to free vorticity, which is the summation of the inflow states $\lambda$ as described in [22] and given by

$$
\begin{aligned}
\dot{\lambda} & =F_{1}\left\{\begin{array}{c}
\ddot{\varepsilon} \\
\dot{\beta}
\end{array}\right\}+F_{2}\left\{\begin{array}{l}
\dot{\varepsilon} \\
\beta
\end{array}\right\}+F_{3} \lambda \\
& =\left[\begin{array}{ll}
F_{1 F} & F_{1 B}
\end{array}\right]\left\{\begin{array}{l}
\ddot{\varepsilon} \\
\dot{\beta}
\end{array}\right\}+\left[\begin{array}{ll}
F_{2 F} & F_{2 B}
\end{array}\right]\left\{\begin{array}{l}
\dot{\varepsilon} \\
\beta
\end{array}\right\}+F_{3} \lambda
\end{aligned}
$$

The aerodynamic loads about the midchord (as defined previously) will be transferred to the wing elastic axis and rotated into the body frame for the solution of the equations of motion. To transfer the loads, one may use

$$
l_{\mathrm{ra}}=l_{\mathrm{mc}} \quad m_{\mathrm{ra}}=m_{\mathrm{mc}}+d l_{\mathrm{mc}} \quad d_{\mathrm{ra}}=d_{\mathrm{mc}}
$$

Furthermore, the aerodynamic loads are rotated as

$$
F^{\text {aero }}=C^{B a_{1}}\left\{\begin{array}{c}
0 \\
d_{\text {ra }} \\
l_{\text {ra }}
\end{array}\right\} \quad M^{\text {aero }}=C^{B a_{1}}\left\{\begin{array}{c}
m_{\mathrm{ra}} \\
0 \\
0
\end{array}\right\}
$$

where $C^{B a_{1}}$ is the transformation matrix from the local aerodynamic frame to the body frame. This matrix is determined by using the
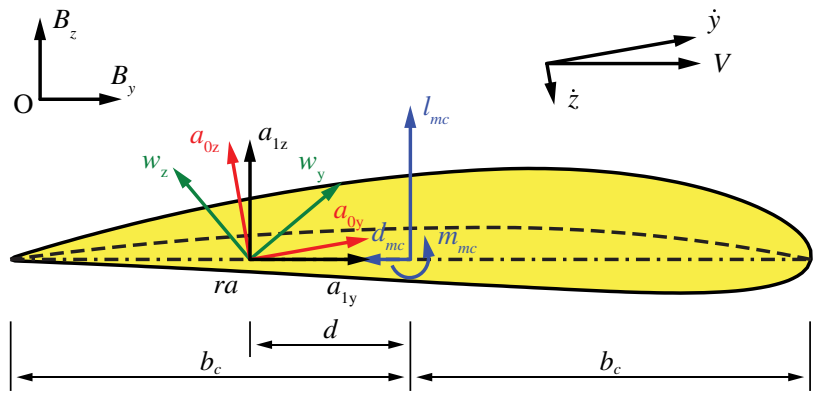

Fig. 2 Airfoil coordinate systems and velocity components. 
instantaneous nodal orientations and has to be updated from the kinematics at each solution step and substep. Note that the finite-span corrections are also included in the force distribution and may come from a computational fluid dynamics solution of the problem or experimental data if available.

\section{F. Equations of Motion with Rigid-Body Degrees of Freedom}

For a beam with free rigid-body motions, one may need additional equations to describe the propagation of its body reference frame. This is particularly important in the coupled nonlinear aeroelastic and flight dynamic analysis. The complete system equations of motion are obtained by augmenting the equations of rigid-body motion and elastic deformations with the inflow equations, which can be represented as

$$
\begin{aligned}
& {\left[\begin{array}{ll}
M_{F F}(\varepsilon) & M_{F B}(\varepsilon) \\
M_{B F}(\varepsilon) & M_{B B}(\varepsilon)
\end{array}\right]\left\{\begin{array}{l}
\ddot{\varepsilon} \\
\dot{\beta}
\end{array}\right\}+\left[\begin{array}{ll}
C_{F F}(\dot{\varepsilon}, \varepsilon, \beta) & C_{F B}(\dot{\varepsilon}, \varepsilon, \beta) \\
C_{B F}(\dot{\varepsilon}, \varepsilon, \beta) & C_{B B}(\dot{\varepsilon}, \varepsilon, \beta)
\end{array}\right]\left\{\begin{array}{l}
\dot{\varepsilon} \\
\beta
\end{array}\right\}} \\
& +\left[\begin{array}{cc}
K_{F F} & 0 \\
0 & 0
\end{array}\right]\left\{\begin{array}{l}
\varepsilon \\
b
\end{array}\right\}=\left\{\begin{array}{l}
R_{F}(\ddot{\varepsilon}, \dot{\varepsilon}, \varepsilon, \dot{\beta}, \beta, \lambda, \zeta) \\
R_{B}(\ddot{\varepsilon}, \dot{\varepsilon}, \varepsilon, \dot{\beta}, \beta, \lambda, \zeta)
\end{array}\right\} \\
& \dot{\zeta}=-\frac{1}{2} \Omega_{\zeta}(\beta) \zeta \quad \dot{P}_{B}=\left[\begin{array}{ll}
C^{G B}(\zeta) & 0
\end{array}\right] \beta \quad \dot{\lambda}=F_{1}\left\{\begin{array}{l}
\ddot{\varepsilon} \\
\dot{\beta}
\end{array}\right\}+F_{2}\left\{\begin{array}{l}
\dot{\varepsilon} \\
\beta
\end{array}\right\}+F_{3} \lambda
\end{aligned}
$$

where $\zeta$ are the quaternions describing the orientation of the body frame $B, P_{B}$ is the inertial position of the $B$ frame, and $C^{G B}$ is the rotation matrix from the body frame to the global frame $G[\underline{3}]$.

\section{G. Modal Solution of the Equations of Motion}

The strain field along the beam coordinate $s$ is approximated by the combination of linear normal modes:

$$
\varepsilon(s, t)=\Phi(s) \eta(t)
$$

where $\Phi$ is the matrix of linear normal strain modes of the beam, and $\eta$ is the corresponding column matrix of the generalized modal coordinate. To obtain the normal modes in strain, one may first assume the modes in the classic displacement form and then derive the mode shapes in strain by taking additional spatial derivatives. This is only applicable for simple beam configurations. A more convenient approach is to use the strain-based finite element equation [Eq. (10)] and perform an eigenvalue analysis with the stiffness and inertia matrices. As the stiffness matrix in Eq. (10) is singular, one can find six zero eigenvalues, which correspond to the free-free rigidbody modes. The remaining eigenvalues are the frequencies for the coupled elastic and rigid-body modes. For the eigenvectors of these coupled modes, they generally take the following form:

$$
\Phi_{C}=\left\{\begin{array}{l}
\Phi_{F} \\
\Phi_{B}
\end{array}\right\}
$$

where $\Phi_{F}$ and $\Phi_{B}$ are the elastic and six-by-one rigid-body components of the modes, respectively. Since the modal approximation in Eq. (18) only requires the elastic deformation, the rigid-body component of these modes is removed, i.e.,

$$
\Phi(s)=\Phi_{F}
$$

After all, if a cantilever beam is to be solved, one only needs to take the elastic portion of the stiffness and inertial matrices (i.e., $K_{F F}$ and $M_{F F}$ ) into the eigenvalue analysis, which yields the modes that satisfy the cantilever boundary condition. One more note about the normal modes is that they are not necessarily obtained about the undeformed shape. One can find normal modes about a geometrically nonlinear deformation. In doing so, the Jacobian matrix from Eq. (11) should be evaluated about the nonlinear deformation, resulting in a different inertia matrix than the undeformed shape. However, the stiffness matrix remains the same.
After the normal modes are obtained, a modal transformation on the equations of motion [Eq. (17)] can be performed based on Eq. (18), i.e.,

$$
\begin{aligned}
& \bar{M}_{F F} \ddot{\eta}+\bar{M}_{F B} \dot{\beta}+\bar{C}_{F F} \dot{\eta}+\bar{C}_{F B} \beta+\bar{K}_{F F} \eta=\bar{R}_{F}(\eta, \dot{\eta}, \ddot{\eta}, \beta, \dot{\beta}) \\
& \bar{M}_{B F} \ddot{\eta}+\bar{M}_{B B} \dot{\beta}+\bar{C}_{B F} \dot{\eta}+\bar{C}_{B B} \beta=\bar{R}_{B}(\eta, \dot{\eta}, \ddot{\eta}, \beta, \dot{\beta}) \\
& \dot{\zeta}=-\frac{1}{2} \Omega_{\zeta}(\beta) \zeta \quad \dot{P}_{B}=\left[\begin{array}{ll}
C^{G B}(\zeta) & 0
\end{array}\right] \beta \\
& \dot{\lambda}=\left[\begin{array}{ll}
\bar{F}_{1 F} & F_{1 B}
\end{array}\right]\left\{\begin{array}{l}
\ddot{\eta} \\
\dot{\beta}
\end{array}\right\}+\left[\begin{array}{ll}
\bar{F}_{2 F} & F_{2 B}
\end{array}\right]\left\{\begin{array}{l}
\dot{\eta} \\
\beta
\end{array}\right\}+F_{3} \lambda
\end{aligned}
$$

where

$$
\begin{aligned}
& \bar{M}_{F F}=\Phi^{T} M_{F F} \Phi \quad \bar{M}_{F B}=\Phi^{T} M_{F B} \quad \bar{M}_{B F}=M_{B F} \Phi \quad \bar{M}_{B B}=M_{B B} \\
& \bar{C}_{F F}=\Phi^{T} C_{F F} \Phi \quad \bar{C}_{F B}=\Phi^{T} C_{F B} \quad \bar{C}_{B F}=C_{B F} \Phi \quad \bar{C}_{B B}=C_{B B} \\
& \bar{K}_{F F}=\Phi^{T} K_{F F} \Phi \\
& \bar{F}_{1 F}=F_{1 F} \Phi \quad \bar{F}_{2 F}=F_{2 F} \Phi
\end{aligned}
$$

The load vector becomes

$$
\begin{gathered}
\left\{\begin{array}{c}
\bar{R}_{F} \\
\bar{R}_{B}
\end{array}\right\}=\left\{\begin{array}{c}
\Phi^{T} K_{F F} \Phi \eta^{0} \\
0
\end{array}\right\}-\left[\begin{array}{c}
\Phi^{T} J_{h \varepsilon}^{T} \\
J_{h b}^{T}
\end{array}\right] N g+\left[\begin{array}{c}
\Phi^{T} J_{p \varepsilon}^{T} \\
J_{p b}^{T}
\end{array}\right] B^{F} F^{\mathrm{dist}} \\
\quad+\left[\begin{array}{c}
\Phi^{T} J_{\theta \varepsilon}^{T} \\
J_{\theta b}^{T}
\end{array}\right] B^{M} M^{\mathrm{dist}}+\left[\begin{array}{c}
\Phi^{T} J_{p \varepsilon}^{T} \\
J_{p b}^{T}
\end{array}\right] F^{\mathrm{pt}}+\left[\begin{array}{c}
\Phi^{T} J_{\theta \varepsilon}^{T} \\
J_{\theta b}^{T}
\end{array}\right] M^{\mathrm{pt}}
\end{gathered}
$$

Finally, the modal solution can be performed based on Eqs. (21-23). Note that the generalized inertia matrix $\bar{M}$, damping matrix $\bar{C}$, and force $\bar{R}$ are all nonlinear and dependent on the Jacobians $J_{h \varepsilon}, J_{p \varepsilon}$, and $J_{\theta \varepsilon}$, which are updated at each time step with subiterations in the numerical integration based on the instantaneous strain vector $\varepsilon$ converted from the modal coordinates $\eta$. This means that the kinematic solution must be performed at each time step. It proved to be a time-consuming process, which will compromise the computational efficiency gained by reducing the dimension of the problem.

\section{Numerical Results}

The strain-based geometrically nonlinear beam formulation has been implemented in the numerical framework named the University of Michigan's Nonlinear Aeroelastic Simulation Toolbox (UM/ NAST). Different solutions can be performed with the finite element approach $[3-5,12,19,20]$, including the geometrically nonlinear static solution, nonlinear transient solution with forced excitations, linear modal solution, stability (flutter) analysis, etc. According to the formulation introduced in the previous section, the nonlinear static and transient solutions are implemented with the modal approach. Then, these two types of analyses will be carried out to study different geometrically nonlinear behaviors of slender beams with different load conditions. In addition, some verification results are provided with respect to displacement-based MSC.Nastran solutions [23] .

\section{A. Transient Solution of a Slender Cantilever Beam}

Consider a slender cantilever isotropic beam (Fig. 3), for which the geometric and physical properties are listed in Table 1 . As shown, a sinusoidal vertical (dead) force is applied at the tip, given by

$$
F(t)=\left\{\begin{array}{cc}
0 & (t<0) \\
A_{F} \sin \omega_{F} t & (t \geq 0)
\end{array}\right.
$$

with $A_{F}=30 \mathrm{~N}$, and $\omega_{F}=20 \mathrm{rad} / \mathrm{s}$. The gravity force is not included in this particular case. The beam is solved in UM/NAST and MSC.Nastran with the beam discretized into 20 elements. The CBEAM element is selected in the MSC.Nastran model. For the modal solutions in UM/NAST, only the flatwise bending modes are 
considered, since the excitation is only in the vertical plane $z x$. The flatwise bending modes and frequencies are obtained from the eigenproblem defined by the finite element approach. Table $\underline{2}$ summarizes the frequencies of the first five flatwise bending modes.

Figure 4 compares the normalized axial and vertical tip displacement results (as the percentage of the beam span) from the different solutions. Basically, by selecting the first bending mode of the beam, one can almost recover (light dashed line in the plot) the finite element solutions from both MSC.Nastran and UM/NAST, except for the discrepancies at some peak values (about 1.4\% relative difference). Additionally, this can be overcome by adding more modes in the solution. With two and three bending modes, the maximum relative errors are about $0.3 \%$ (two modes) and $0.1 \%$ (three modes), respectively. Figure 5 illustrates the maximum vertical displacements of the nodes along the beam span. Results from different solutions are compared. One can only observe a very slight difference between the finite element method (FEM) (solid line) and the one-mode (square markers) results, whereas the two-mode and three-mode results are almost sitting on top of the FEM result.

Table 3 lists the problem size (in number of degrees of freedom) and $\mathrm{CPU}$ time (normalized with respect to the time of the finite element solution) of the four solutions. It is evident that the complexity of the modal solutions is significantly reduced compared to the finite element solution. In addition, modal solutions are more efficient than the finite element solution, with over $24 \%$ reduction on CPU time. However, when varying the number of modes involved in the modal solutions, one cannot see prominent changes in the computing time, due to the added cost for the kinematic solutions from the modal amplitudes, as discussed before.

\section{B. Transient Response of Slender Beam with Rigid-Body Motion (Free Boundary Condition)}

The cantilever beam is now extended to a span of $6 \mathrm{~m}$, whereas the cross-sectional properties are kept unchanged. There is no rigid-body constraint applied to the beam. Sinusoidal vertical forces governed by Eq. (24) are applied at the locations indicated in Fig. 6 , with the amplitudes of $F_{0}$ to $F_{4}$ being $44,-34,-34,12$, and $12 \mathrm{~N}$, respectively. The frequencies of these excitations are all $5 \mathrm{~Hz}$. The loads are so defined such that their summation at any time is zero. Transient response of the beam with such loading and boundary conditions is obtained with both finite element and modal approaches in UM/NAST, where the time step used is $0.0025 \mathrm{~s}$.

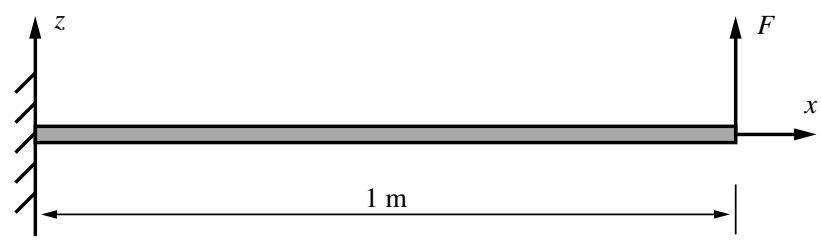

Fig. 3 Cantilever beam with tip load.

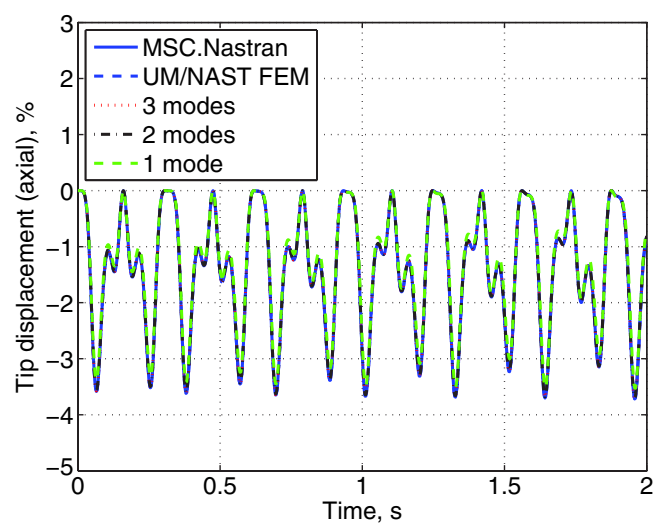

a) Axial displacement
Table 1 Properties of the cantilever isotropic beam

\begin{tabular}{lcc}
\hline \hline Property & Value & Unit \\
\hline Span $L$ & 1.00 & $\mathrm{~m}$ \\
Mass per span $m$ & 0.10 & $\mathrm{~kg} / \mathrm{m}$ \\
Rotational moment of inertia & $1.30 \times 10^{-4}$ & $\mathrm{~kg} \cdot \mathrm{m}$ \\
Flat bending moment of inertia & $5.00 \times 10^{-6}$ & $\mathrm{~kg} \cdot \mathrm{m}$ \\
Edge bending moment of inertia & $1.25 \times 10^{-4}$ & $\mathrm{~kg} \cdot \mathrm{m}$ \\
Extensional rigidity & $1.00 \times 10^{6}$ & $\mathrm{~N}$ \\
Torsional rigidity & 80.0 & $\mathrm{~N} \cdot \mathrm{m}^{2}$ \\
Flat bending rigidity & 50.0 & $\mathrm{~N} \cdot \mathrm{m}^{2}$ \\
Edge bending rigidity & $1.25 \times 10^{3}$ & $\mathrm{~N} \cdot \mathrm{m}^{2}$ \\
\hline \hline
\end{tabular}

Table 2 Natural modes and frequencies of the cantilever isotropic beam

\begin{tabular}{clc}
\hline \hline Number & \multicolumn{1}{c}{ Mode } & Frequency, $\mathrm{Hz}$ \\
\hline 1 & First flat bend & 12.5 \\
2 & Second flat bend & 78.7 \\
3 & Third flat bend & $2.21 \times 10^{2}$ \\
4 & Fourth flat bend & $4.37 \times 10^{2}$ \\
5 & Fifth flat bend & $7.31 \times 10^{2}$ \\
\hline \hline
\end{tabular}

The beam is first divided into 24 elements in UM/NAST to generate the modes with the rigid-body motions (Fig. 7). Note that there are six zero-frequency rigid-body modes associated with the beam, but they are not used in the modal solution. Even for the plots in Fig. 7, only the elastic components of the modes are shown and applied in the modal solution (Eq. 18). In addition, only the symmetric flatwise bending modes are considered due to the specific loading condition defined for the beam.

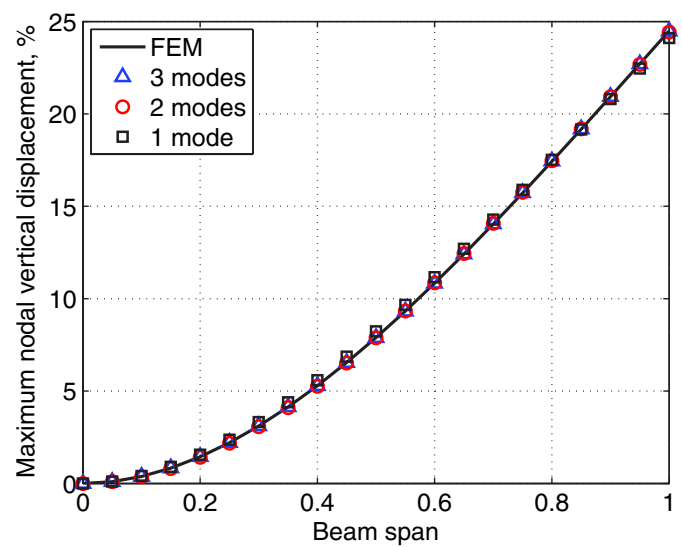

Fig. 5 Maximum vertical displacement of nodes along the beam, normalized with respect to beam span.

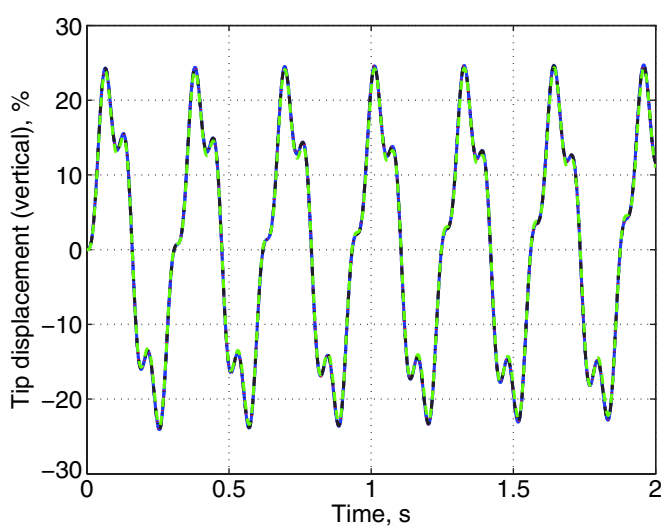

b) Vertical displacement

Fig. 4 Tip displacement of the cantilever beam, normalized with respect to beam span. 
Table 3 Problem size and normalized computing time of different solutions for the cantilever beam case

\begin{tabular}{lcc}
\hline \hline Solution & Dimension & CPU time \\
\hline FEM & 80 & 1 \\
Modal: one mode & 1 & 0.744 \\
Modal: two modes & 2 & 0.756 \\
Modal: three modes & 3 & 0.758 \\
\hline \hline
\end{tabular}

Figure $\underline{8}$ shows the vertical displacements at the stations at two thirds of the span (points A and B, see Fig. 6) and the tip (points $\mathrm{C}$ and D, see Fig. 6) of the beam. With the loads defined for the beam, nodal displacements lie in the vertical plane determined by the loads. Obviously, with only the first flatwise bending mode, it is not sufficient to accurately model the beam deformation. The loading is so applied to excite the second flatwise bending mode. Therefore, at least two modes are required to represent the beam motion. One may compare the modal solutions with two and three modes versus the finite element results. The two-mode modal solution still deviates from the finite element solution at some peak values, whereas the

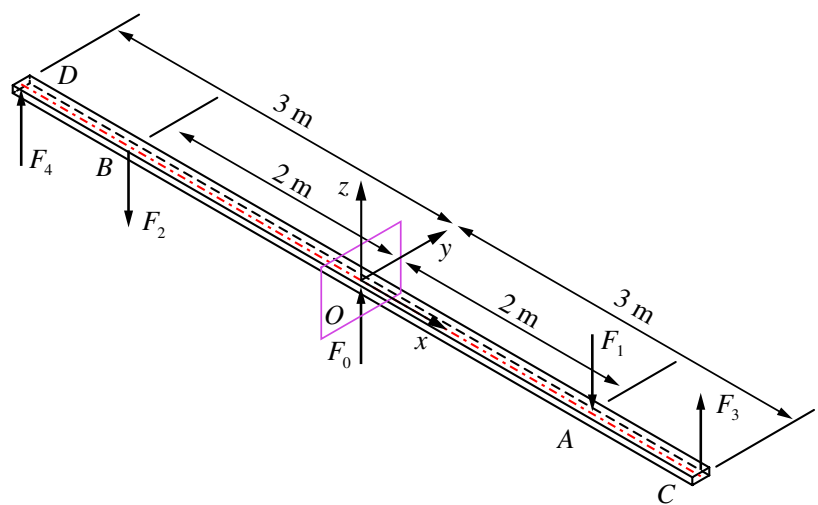

Fig. 6 Flexible beam with multipoint excitations and free boundary condition. solution with three modes converges well to the finite element solution. This can also be observed from the maximum vertical displacements of nodes along the beam span (Fig. 9).

As no rigid-body constraint is applied to the beam, it is also of interest to analyze its rigid-body motion. Since under large deformations the c.g. of the structure will be varying with deformation, it is convenient to select a point that will become the origin of the body-fixed reference frame about which the rigid-body motions are defined. There is not a unique choice, and even the c.g. of the undeformed configuration can be selected. For this particular example, the beam middle point was selected about which the rigidbody motion of the beam is defined. Figure 10 plots the vertical displacement and velocity of that reference point. The results indicate that the selection of the modes does impact the solution of the rigidbody motion.

Table 4 compares the problem size and CPU time of the different solutions for this flexible beam. The complexity of the problem to be solved is reduced in modal solutions. Again, due to the recovery of kinematics at each time step and subiterations within one time step, the time used for modal solutions (with two and three modes) is not significantly reduced compared to the finite element method. The

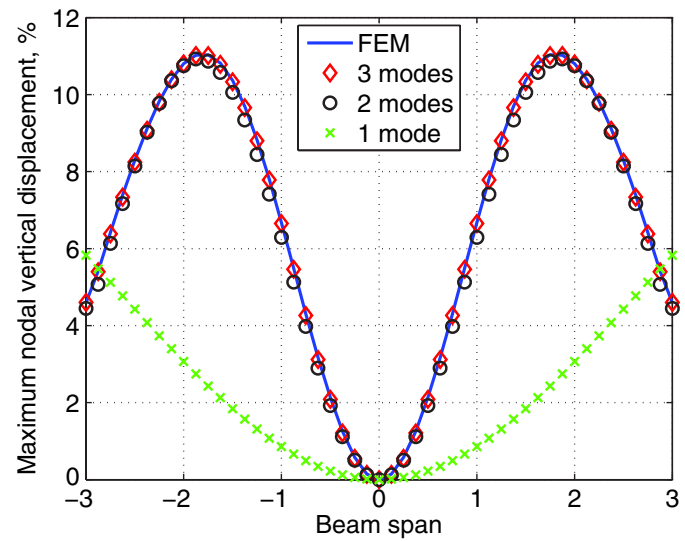

Fig. 9 Maximum vertical displacement of nodes along the beam, normalized with respect to beam half-span.

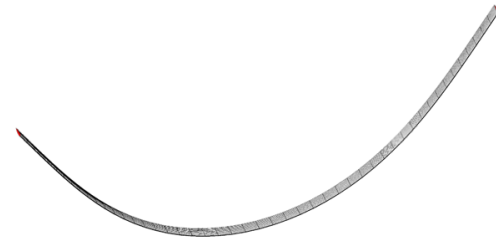

a) Mode 1: $2.22 \mathrm{~Hz}$

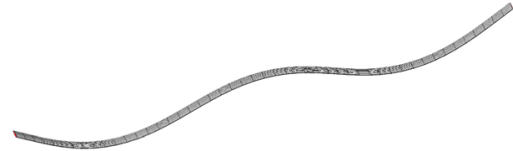

b) Mode 2: $12.1 \mathrm{~Hz}$

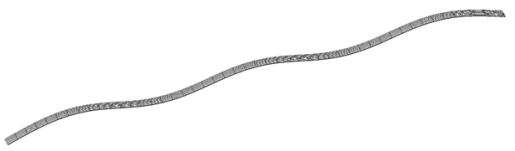

c) Mode 3: $30.4 \mathrm{~Hz}$

Fig. 7 First three symmetric flatwise bending modes of the free flexible beam.

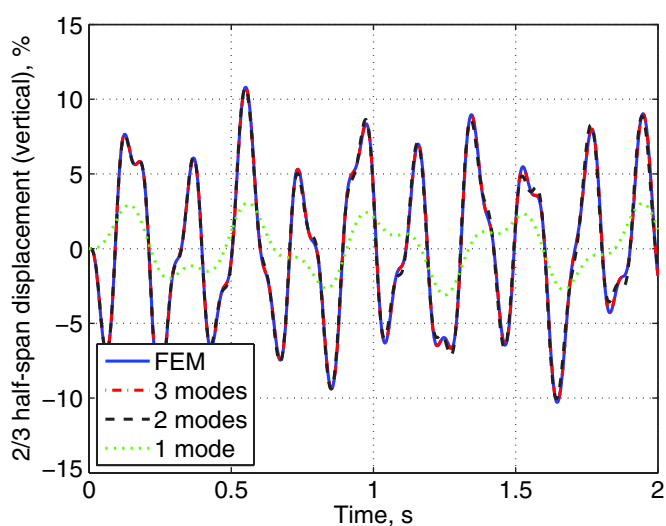

a) Two-thirds of half-beam span

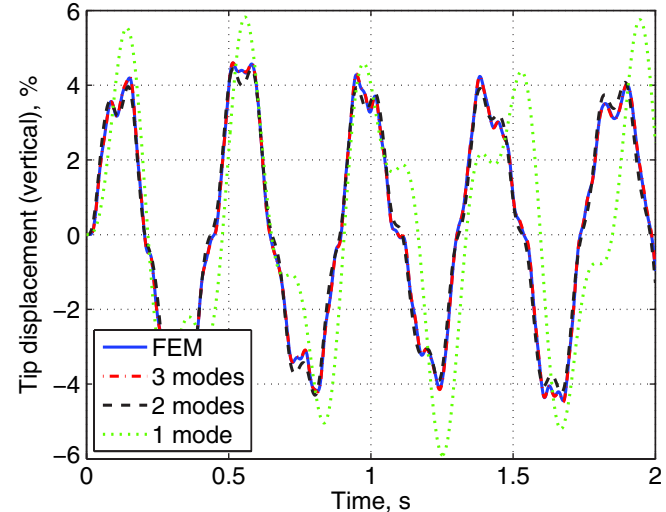

b) Beam tip

Fig. 8 Beam vertical displacements due to elastic deformation, normalized with respect to beam half-span. 


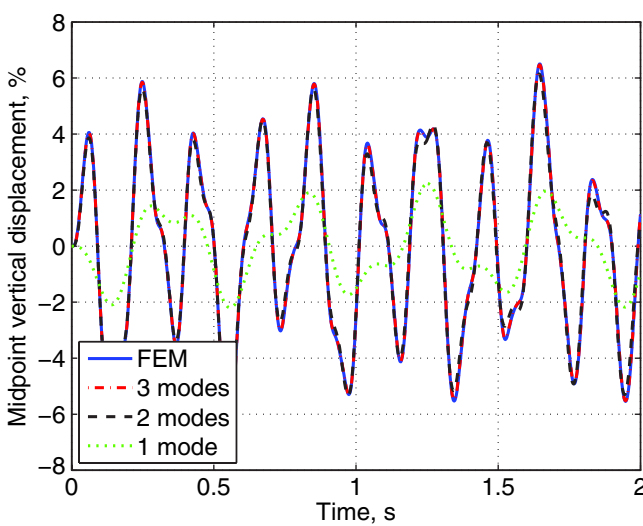

a) Body frame displacement

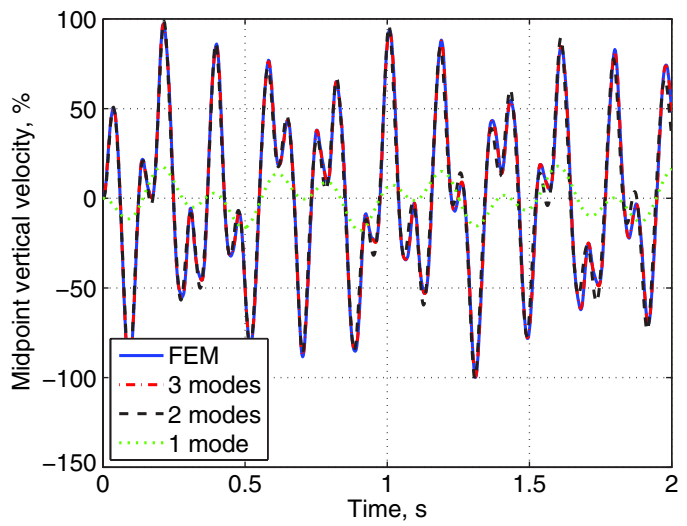

b) Body frame velocity

Fig. 10 Middle-point (body frame) vertical displacement and velocity, with displacement normalized with respect to beam half-span and velocity normalized with respect to its maximum value $(7.12 \mathrm{~m} / \mathrm{s})$.

large reduction in the CPU time of the modal solution with one mode is due to the fact that fewer subiterations are required to converge at each time step than the other two cases.

\section{Description of a Highly Flexible Wing}

In [1], a highly flexible high-aspect-ratio wing was used for aeroelastic analyses. The physical and geometrical properties of the wing are given in Table 5. The natural frequencies and linear flutter speed $\$$ of this model were calculated in [1]. Natural modes are calculated for the undeformed beam using the finite element approach in the current formulation. The first few natural frequencies are listed in Table 6. An eight-mixed-based finite element discretization was used in [1], whereas 10 and 20 strain-based elements are employed in the current discretization for convergence studies. These results are all compared with analytical solutions. As can be observed, the current formulation gives accurate numerical predictions on the fundamental frequencies of the slender beam when compared to the analytical solutions.

Using the strain-based formulation, the linear flutter result at the $20,000 \mathrm{~m}$ altitude without gravity effects is obtained and compared with what was presented in [1] (see Table 7). The current strain-based formulation predicts identical linear results as in [1]. In addition, the nonlinear flutter boundary (root pitch angle $=2 \mathrm{deg}$ ) is also calculated with this cantilever wing (see Table 7). The flutter mode is a coupled second flatwise bending and first torsion, whereas the inplane bending component in the flutter mode is not significant (see Fig. 11). Note that the flutter boundaries are obtained using the approach introduced in [4], where the finite element solution of the system equations was involved. In the coming sections, simulations are performed to study the static deformation and postflutter behavior of the flexible cantilever wing by using the modal analysis.

\section{Static Aeroelastic Solution of the Highly Flexible Wing}

To assess the convergence of the static modal solutions for the aeroelastic problem, a series of calculations are performed to obtain the static deformations of the flexible wing with different numbers of modes. The freestream velocity is $25 \mathrm{~m} / \mathrm{s}$ (greater than its nonlinear flutter boundary), and the root pitch angle is $2 \mathrm{deg}$ (the same as the one used for the nonlinear flutter calculation). Figures 12a, 13a, and 14 a exemplify the convergence trend when more modes are involved in the calculation, where the solid lines in the plots represent the results of the finite element solution with 20 elements (the reference solution). In the modal solutions, the modes are selected from the list in Table $\underline{6}$ with 20 -element discretization. As one can observe from the plots, the modal solution including the first three modes is sufficiently accurate since the relative errors of the tip displacements

\$Linear flutter" means the instability of the numerical system is found based on the undeformed wing configuration; it is not the actual wing flutter boundary due to geometrically nonlinear effects. in the three directions are all less than $2 \%$. A more accurate solution can be obtained with seven modes included, where the relative error of the tip displacements is within $0.5 \%$.

Another set of static modal solutions is conducted using the mode shapes about a beam deformed shape. Particularly, the deformation under loads generated from a $25 \mathrm{~m} / \mathrm{s}$ freestream velocity is obtained (using FEM), about which another set of mode shapes is obtained (see the Appendix for the plots of these modes). Based on this new set of modes, the static deformation could be approximated again and compared to the finite element results. From these, it can be found that the convergence of the solution using modes about the deformed shape is faster than the one using the undeformed shape. To reach the same $0.5 \%$ relative error, only five modes are required in the solution (see Figs. 12b, 13b, and 14b).

\section{E. Limit-Cycle Oscillation of the Highly Flexible Wing}

In this section, the transient response of the highly flexible wing is analyzed. The wing is first brought to its nonlinear static deformation with the freestream velocity of $25 \mathrm{~m} / \mathrm{s}$, calculated in the previous section. The deformed shape serves as the initial condition for the transient simulations. Both 20-finite-element and modal simulation cases (see Table 8) are solved. Note that the modes used for the modal solutions are selected from the list of Table $\underline{6}$, which are obtained about the undeformed state. As the freestream velocity is higher than the flutter boundary $(23.3 \mathrm{~m} / \mathrm{s})$, the wing is expected to self-excite.

Table 4 Problem size and normalized computing time of different solutions for the free flexible beam case

\begin{tabular}{lccc}
\hline \hline & \multicolumn{3}{c}{ Dimension } \\
Solution & Elastic & Rigid body & CPU time \\
\hline FEM & 96 & 6 & 1 \\
Modal: one mode & 1 & 6 & 0.626 \\
Modal: two modes & 2 & 6 & 0.913 \\
Modal: three modes & 3 & 6 & 0.939 \\
\hline \hline
\end{tabular}

Table 5 Properties of a highly flexible wing (after [1] $)$

\begin{tabular}{lcc}
\hline \hline Property & Value & Unit \\
\hline Span $L$ & 16 & $\mathrm{~m}$ \\
Chord $c$ & 1 & $\mathrm{~m}$ \\
Beam reference axis (from LE) & $50 \%$ chord & $\mathrm{m}$ \\
Cross-sectional c.g. (from LE) & $50 \%$ chord & $\mathrm{m}$ \\
Mass per span $m$ & 0.75 & $\mathrm{~kg} / \mathrm{m}$ \\
Rotational moment of inertia & 0.1 & $\mathrm{~kg} \cdot \mathrm{m}$ \\
Torsional rigidity & $1.00 \times 10^{4}$ & $\mathrm{~N} \cdot \mathrm{m}^{2}$ \\
Flat bending rigidity & $2.00 \times 10^{4}$ & $\mathrm{~N} \cdot \mathrm{m}^{2}$ \\
Edge bending rigidity & $4.00 \times 10^{6}$ & $\mathrm{~N} \cdot \mathrm{m}^{2}$ \\
\hline \hline
\end{tabular}


Table 6 Natural modes and frequencies of the highly flexible wing (in hertz) about its undeformed shape

\begin{tabular}{clcccc}
\hline \hline Number & \multicolumn{1}{c}{ Mode } & Ref. [1] & $\begin{array}{c}\text { Strain-based beam } \\
(10 \text { elements })\end{array}$ & $\begin{array}{c}\text { Strain-based beam } \\
(20 \text { elements })\end{array}$ & $\begin{array}{c}\text { Analytical } \\
\text { solution }\end{array}$ \\
\hline 1 & First flat bend & 0.358 & 0.358 & 0.357 & 0.357 \\
2 & Second flat bend & 2.33 & 2.27 & 2.25 & 2.24 \\
3 & First torsion & 4.96 & 4.95 & 4.94 & 4.94 \\
4 & First edge bend & 5.05 & 5.06 & 5.05 & 5.05 \\
5 & Third flat bend & 7.01 & 6.53 & 6.33 & 6.26 \\
6 & Fourth flat bend & - & 13.3 & 12.5 & 12.3 \\
7 & Second torsion & - & 15.0 & 14.9 & 14.8 \\
8 & Fifth flat bend & - & 23.2 & 21.0 & 20.3 \\
9 & Third torsion & - & 25.3 & 24.9 & 24.7 \\
10 & Second edge bend & - & 32.2 & 31.8 & 31.6 \\
\hline \hline
\end{tabular}

Table 7 Flutter boundary of the highly flexible wing

\begin{tabular}{lccc}
\hline \hline & Ref. [1] & Current (linear) & Current (nonlinear) \\
\hline Velocity, $\mathrm{m} / \mathrm{s}$ & 32.2 & 32.2 & 23.3 \\
Frequency, Hz & 3.60 & 3.60 & 1.61 \\
\hline \hline
\end{tabular}

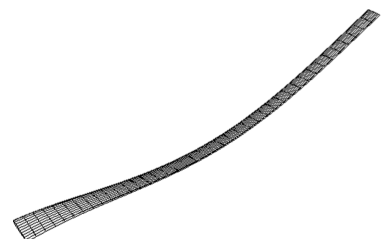

Fig. 11 Flutter mode of the flexible wing (left: axonometric view; right: front view).
The amplitude of the wing oscillation would increase until it reaches the limit-cycle oscillation (LCO).

Figure $15 \mathrm{a}$ shows the time history (from 0 to $100 \mathrm{~s}$ ) of the tip vertical displacement. Note that the quantities are normalized by the wingspan. For clarity purposes, profile contours for cases 3 to 5 are provided in this plot (Fig. 15a), such that the reference finite element results can be clearly observed. Figures $15 \mathrm{~b}$ and $15 \mathrm{c}$ give the transient response when the LCO is still developing, whereas Fig. $15 \mathrm{~d}$ exemplifies the steady state of the LCO. A phase-plane plot of the wingtip motion is provided in Fig. 16. In addition, one can take the 20 periods of the wingtip displacement after $85 \mathrm{~s}$, when the LCO is set, and perform a fast Fourier transform (FFT) to obtain the frequency components of the response, which are plotted in Fig. 17. From Figs. 16 and 17 , one can basically see a one-frequency LCO of this aeroelastic system.

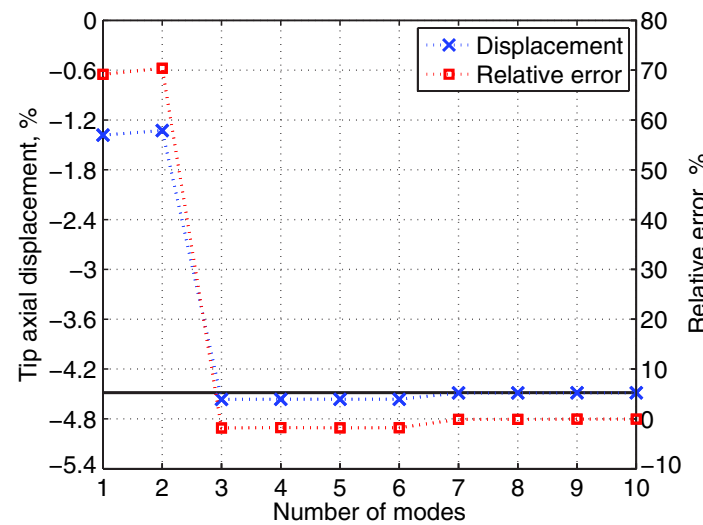

a) Calculation using modes about the undeformed shape

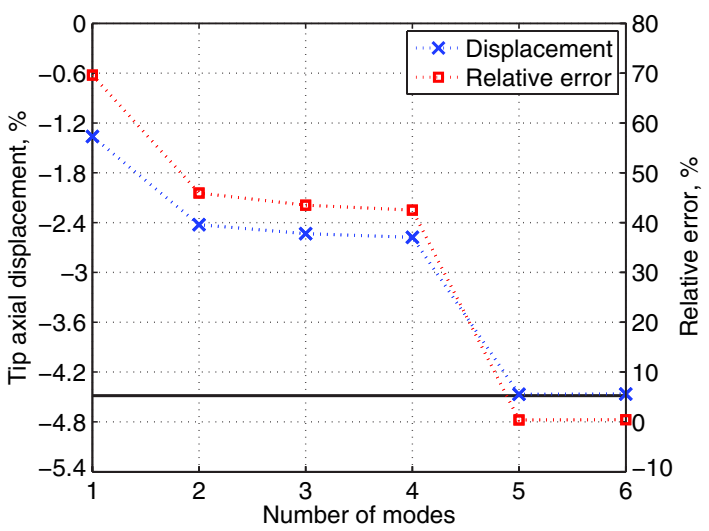

b) Calculation using modes about the deformed state with $25 \mathrm{~m} / \mathrm{s}$ freestream velocity

Fig. 12 Change of the steady-state tip axial displacement with number of modes.

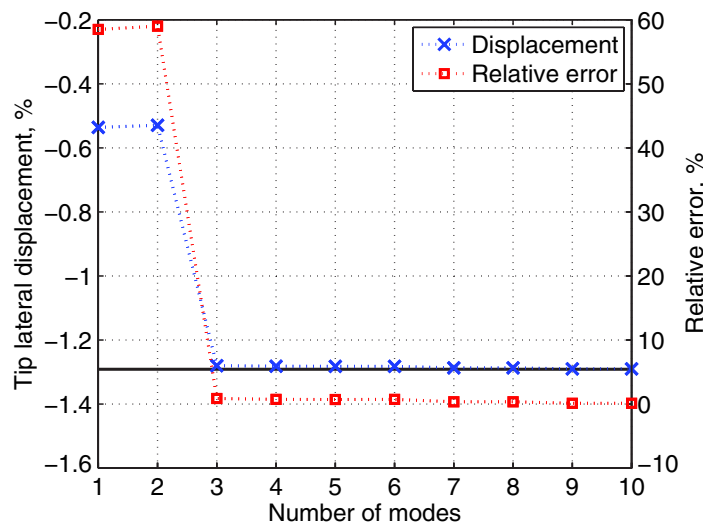

a) Calculation using modes about the undeformed shape

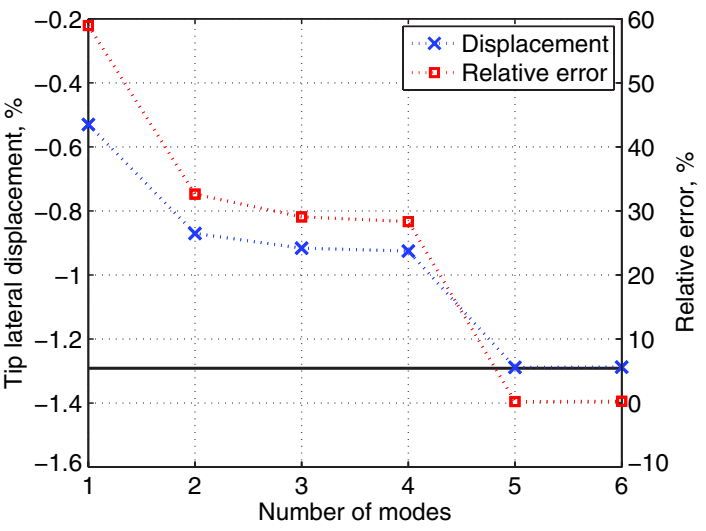

b) Calculation using modes about the deformed state with $25 \mathrm{~m} / \mathrm{s}$ freestream velocity

Fig. 13 Change of the steady-state tip lateral displacement with number of modes. 


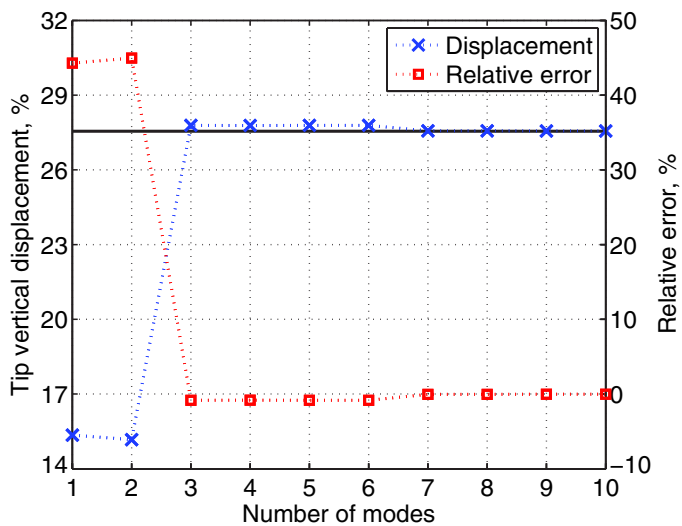

a) Calculation using modes about the undeformed shape

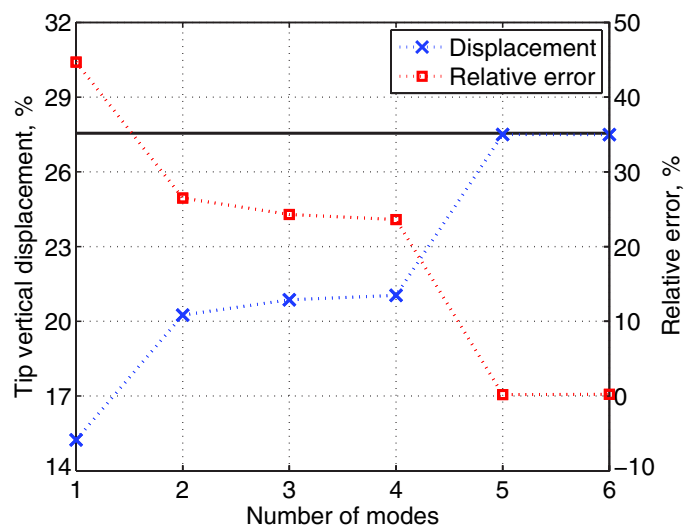

b) Calculation using modes about the deformed state with $25 \mathrm{~m} / \mathrm{s}$ freestream velocity

Fig. 14 Change of the steady-state tip vertical displacement with number of modes.

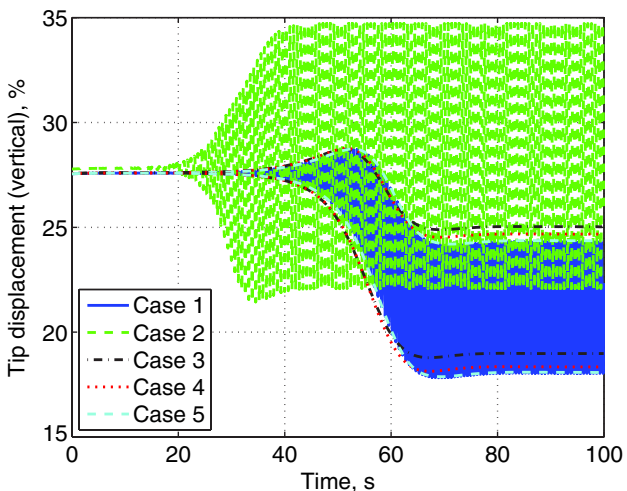

a) Time: 0-100 s (contours of Cases 3 to 5 are provided)

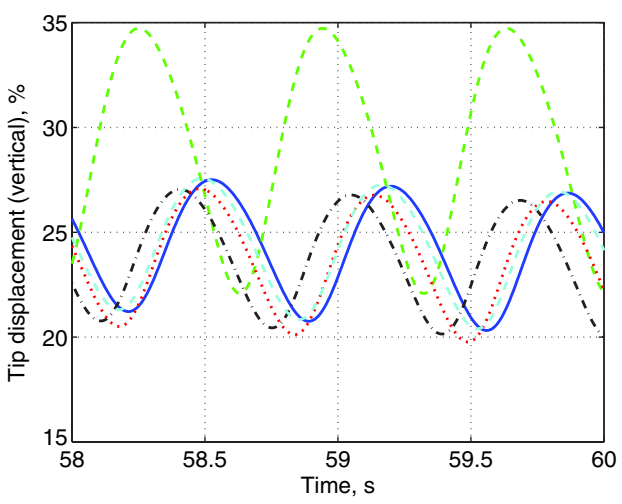

c) Time: 58-60 s

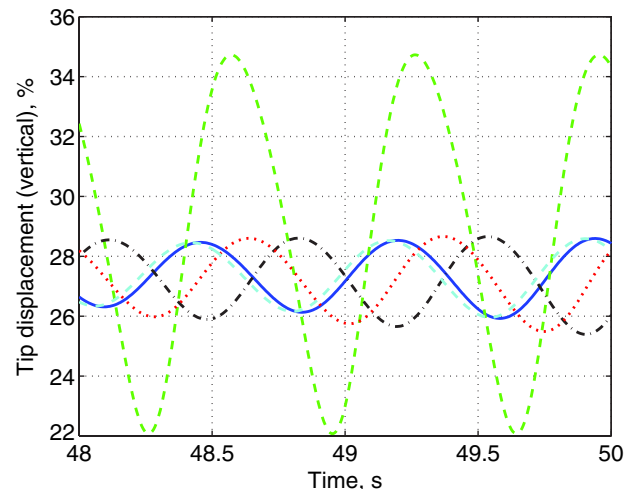

b) Time: $48-50 \mathrm{~s}$

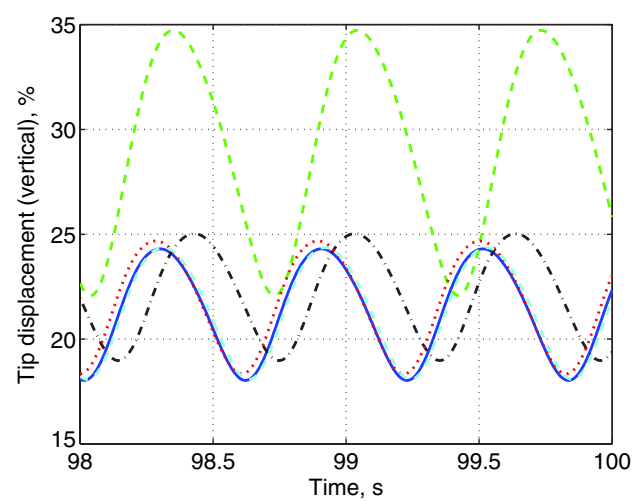

d) Time: 98-100 s

Fig. 15 LCO of the highly flexible wing: solution with normal modes about the undeformed shape.

As shown in the figures, with the selection of the first four modes (case 2: the one with larger oscillation amplitude), it is possible to predict the instability of the aeroelastic system. The transient response eventually develops into an LCO. However, its magnitude (Fig. 15), phase (Fig. 16), and frequency (Fig. 17) do not agree with the reference finite element simulation at all, as a completely different LCO behavior is predicted. Note that it was already sufficient to use only the first four modes to provide converged static solutions (see Figs. 12-14). Therefore, it is necessary to include more modes in the transient analysis to converge the solution. That is how the study is performed in this work, as cases 3 to 5 are carried out with 7, 9, and 15 modes, respectively. Obviously, the convergence gets better with more modes involved; and with 15 modes, the modal analysis results agree with those from the finite element analysis. From this analysis, one can conclude that more modes in modal transient analysis is
Table 8 Transient analysis cases of the highly flexible wing with normal modes about the undeformed shape

\begin{tabular}{cc}
\hline \hline Case number & Description \\
\hline 1 & FEM \\
2 & Modes 1-4 \\
3 & Modes 1-7 \\
4 & Modes 1-9 \\
5 & Modes 1-15 \\
\hline \hline
\end{tabular}




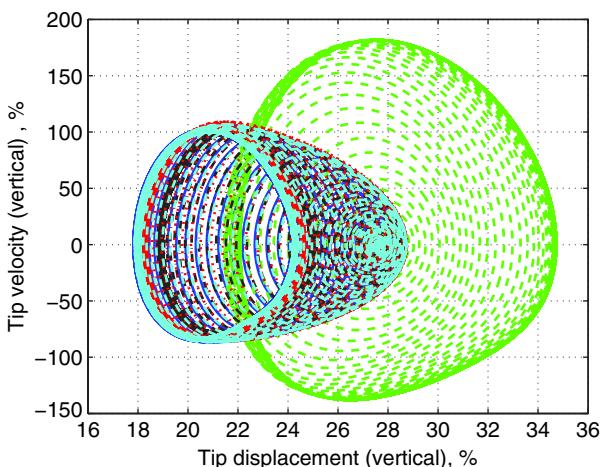

a) Time: 0-100 s

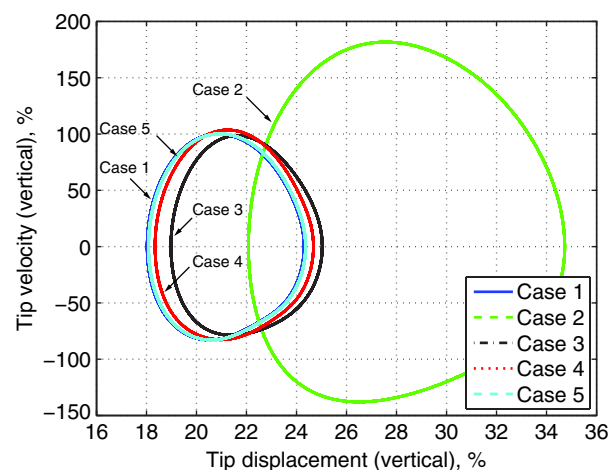

b) Time: $85-100 \mathrm{~s}$

Fig. 16 Phase-plane plot of the wingtip motion, displacement normalized by wing span, and velocity normalized by the maximum value from the FEM solution $(6.27 \mathrm{~m} / \mathrm{s})$.

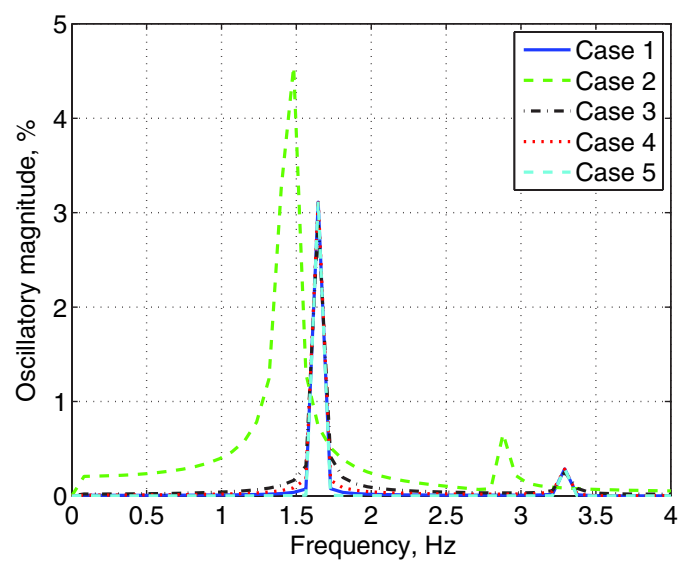

Fig. 17 FFT of the tip oscillation of the highly flexible wing ( 20 periods after 85 s): amplitude normalized by wingspan.

needed than the static solution, as expected for this aeroelastic problem.

Table 9 still compares the problem size and the CPU time for the different solutions of the aeroelastic problem. In the simulations, the wing is divided into 20 stations, on each of which the inflow velocity is calculated with the expansion of six inflow states. Therefore, the

Table 9 Problem size and normalized computing time of different transient solutions of the highly flexible wing with normal modes about the undeformed shape

\begin{tabular}{lrcc}
\hline \hline & \multicolumn{2}{c}{ Dimension } & \\
\cline { 2 - 3 } Solution & Elastic & Aerodynamic & CPU time \\
\hline Case 1 & 80 & 120 & 1 \\
Case 2 & 4 & 120 & 1.065 \\
Case 3 & 7 & 120 & 1.084 \\
Case 4 & 9 & 120 & 1.100 \\
Case 5 & 15 & 120 & 1.152 \\
\hline \hline
\end{tabular}

Table 10 Transient analysis cases of the highly flexible wing with normal modes about the deformed shape

\begin{tabular}{cc}
\hline \hline Case number & Description \\
\hline 1 & FEM \\
2 & Modes 1-4 \\
3 & Modes 1-5 \\
4 & Modes 1-7 \\
5 & Modes 1-9 \\
\hline \hline
\end{tabular}

total number of aerodynamic states is 120 . The current study does not vary the number of strips in the aerodynamic calculation. Further parametric study can be performed to evaluate the impact of different numbers of aerodynamic strips and states in the aeroelastic solution. From the comparison of the CPU time, all solutions are at comparable times, with the modal solutions taking longer than the direct FEM one. This is due to the fact that nodal orientations are recovered in the intermediate steps of the time integrations, in addition to the Jacobians required in the simulations with only structural loads. The nodal orientations are used to determine the local aerodynamic frames (by using $C^{B a 1}$ ), such that the velocity components and angles of attack are obtained.

Similar to the static analysis, the normal modes about the deformed shape can also be used as the reference modes in the transient analysis. Now, the normal modes are determined about the beam deformed shape under $25 \mathrm{~m} / \mathrm{s}$ freestream. Different modal analysis cases are compared to the finite element solution (see Table 10). Again, the time history of the wingtip vertical displacement, the phase-plane plot of the wingtip motion, and the FFT results of the wingtip displacement are plotted in Figs. 18-20, respectively.

Figure 18a plots the time history of the vertical displacement for the wingtip. Profile contours for cases 3 to 5 are provided in this plot, such that the reference finite element results can be clearly observed. Different stages in the time history are also illustrated in Figs. 18b$18 \mathrm{~d}$. It can be observed that, with only four modes (case 2, nonoscillatory), the simulation starts from a different initial (static) condition and does not predict instability at all. A minimum of five modes is required to capture the instability of the system. Figure 19 exemplifies the motion of wingtip with the phase-plane plot. One can clearly see that case 2 behaves differently from all the other cases, which reduces to a point in the phase-plane plot, and no oscillation is observed. From Figs. 18b, 18c, and 19a, the phase difference of the other cases can be observed at the transient stages of the simulations, when the limit-cycle oscillation is still developing. However, after the LCO is set up (Figs. 18d and 19b), the phase difference is reduced and maintains constant. In addition, the magnitude of the oscillations (displacement and velocity) from the modal solutions (cases 3 to 5) is very close to what the finite element solution predicts. From the plot of Fig. 20, the frequency components of the responses in cases 3 to 5 are almost the same as the finite element solution. Therefore, the solution with five modes (case 3, dark dashed-dotted line) is already good enough compared to the finite element result. It has some phase differences from the FEM results, but the magnitude and frequency match well. With more modes included, the convergence quality is slightly, but not significantly, improved. Overall, the simulations using the modes about the deformed shape converge faster compared to the simulations using the normal modes about the undeformed shape. Note that selection of the first five modes about the deformed shape also gave nicely converged static solutions. As the solution time of using the modes about the deformed configuration is comparable to the one about the undeformed shape, the comparison of the CPU times of these cases is not discussed here. 


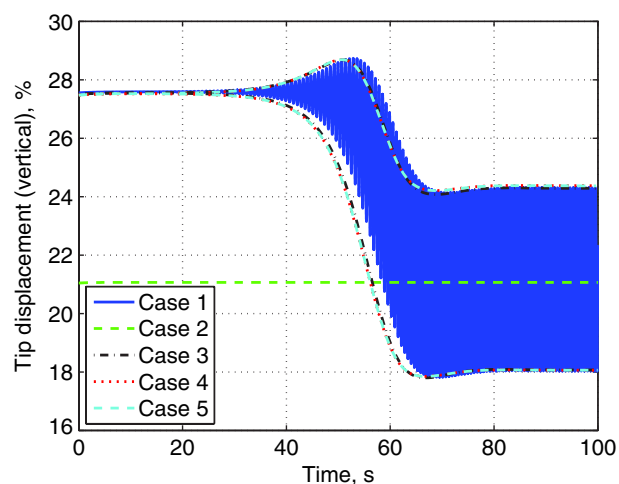

a) Time: 0-100 s (contours of Cases 3 to 5 are provided)

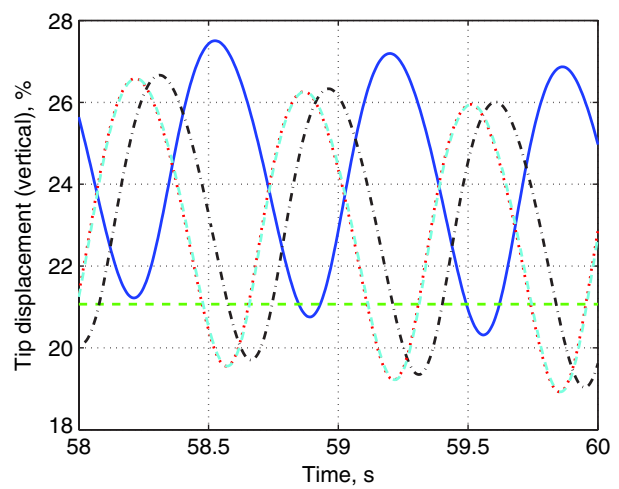

c) Time: $58-60 \mathrm{~s}$

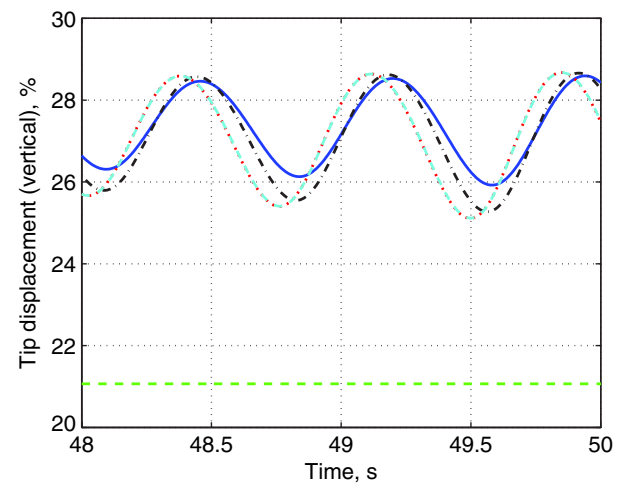

b) Time: $48-50 \mathrm{~s}$

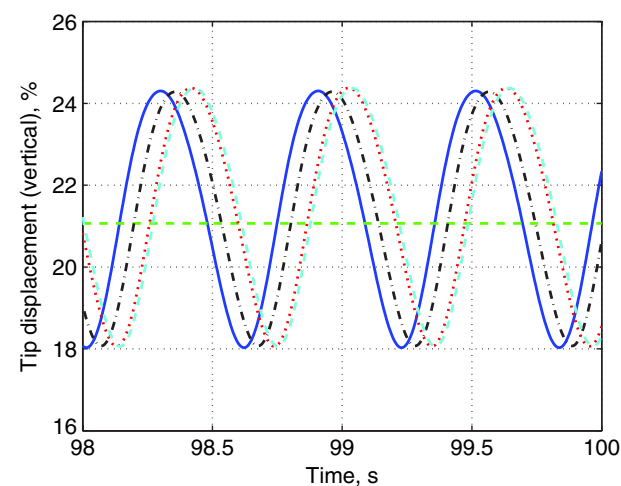

d) Time: 98-100 $\mathrm{s}$

Fig. 18 LCO of the highly flexible wing: solution with normal modes about the deformed shape.

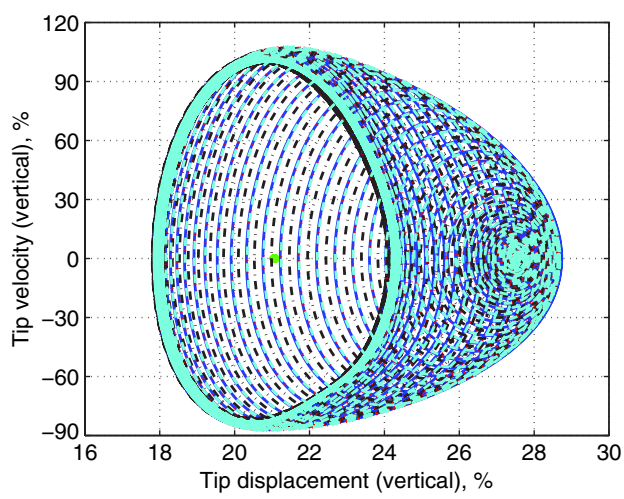

a) Time: $0-100 \mathrm{~s}$

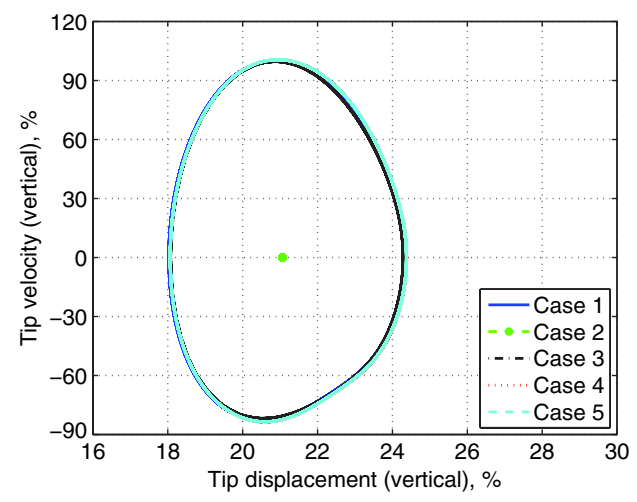

b) Time: 85-100 s

Fig. 19 Phase-plane plot of the wingtip motion, displacement normalized by wingspan, and velocity normalized by the maximum value from the FEM solution $(6.27 \mathrm{~m} / \mathrm{s})$.

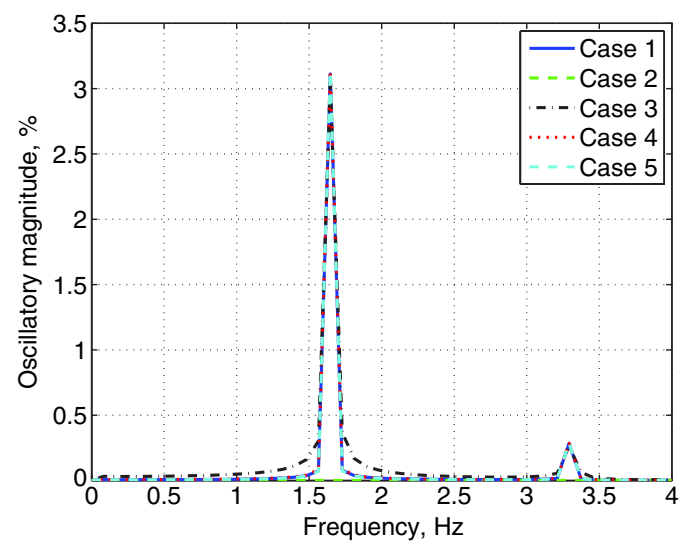

Fig. 20 FFT of tip displacement of the highly flexible wing (20 periods after 85 s): amplitude normalized by wingspan.

\section{Conclusions}

This paper discussed a modal solution to the strain-based geometrically nonlinear beam formulation. The original strain-based geometrically nonlinear beam formulation is able to capture the arbitrarily large displacements of slender structures. The theory defines beam extension strain and bending/twist curvatures as independent states, which makes no approximation to the deformation of beam reference line. Therefore, this formulation is geometrically exact and can accurately model the composite beam deformation. The strainbased formulation features fewer degrees of freedom than the displacement-based and the mix-form formulations to represent the same deformation complexity. In addition, this formulation solves directly for the beam curvatures that are the variables measured by typical sensors in control studies (e.g., strain gauges).

The linear normal modes in strain are used to approximate the strain distribution (also displacement distribution through the recovery of kinematics) along the beam. As one can observe from the 
numerical studies, the major advantage of the modal approximation is the simplicity of the model because of the further reduction of the problem size when compared to the direct finite element form. Another advantage of the strain-based modal solution is its simplicity in finding the geometrically nonlinear static solution. With a few modes involved, one can find out the accurate static solution. Moreover, the generalized modal stiffness matrix is constant, which means it does not need to be updated over iterations to find the converged nonlinear static state. This makes this solution approach very suitable for the trim solution of very flexible aircraft, where the solution is fundamentally static and geometrically nonlinear.

Nonlinear transient analyses were also performed for various beams with prescribed or aerodynamic loads. From the numerical studies, it is verified that the use of normal modes about a geometrically nonlinear deformed shape may improve the convergence of the solution: that is, fewer modes are required to reach the converged solution. This is also of importance in the nonlinear aeroelastic analysis of very flexible aircraft. For that, the geometrically nonlinear trim state should be used as the position about which to extract the normal modes. Transient analysis may be accurately performed by properly choosing few selected modes.

Although it was expected that employing a modal representation for the structural solution would result in lower computational times, the reduction of computational times was not substantial. The computational advantage of the new solution was sacrificed due to the requirement of recovering wing displacement and rotation from kinematics for every aeroelastic time step. Clearly, a better way to handle the intermediate wing displacement/rotation in the time integration is highly desirable to improve the computational efficiency of the overall aeroelastic solution.

\section{Appendix: Normal Modes of the Highly Flexible Wing about Deformed Shape}

Listed in Figs. A1-A10 are the first 10 modes obtained for the cantilever wing about its deformed shape as the result of aerodynamic loading generated from a $25 \mathrm{~m} / \mathrm{s}$ freestream flow. Note that all the torsional modes are coupled with some edgewise and flatwise bending components, which are not legible among the plots.

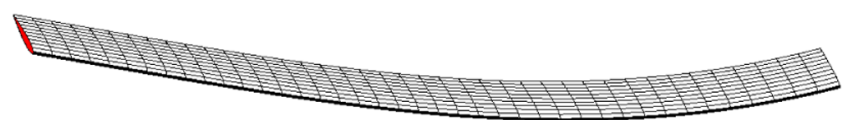

Fig. A1 First mode (first flat bending, $0.358 \mathrm{~Hz}$ ).

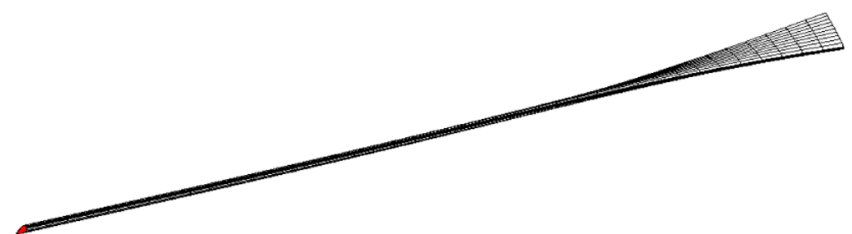

Fig. A2 Second mode (first torsion, $1.49 \mathrm{~Hz}$ ).

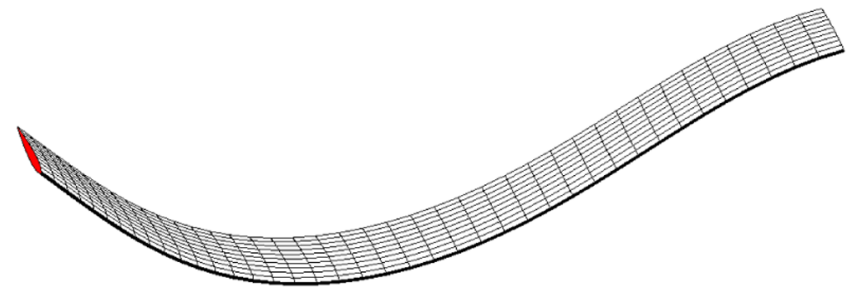

Fig. A3 Third mode (second flat bending, $2.22 \mathrm{~Hz}$ ).

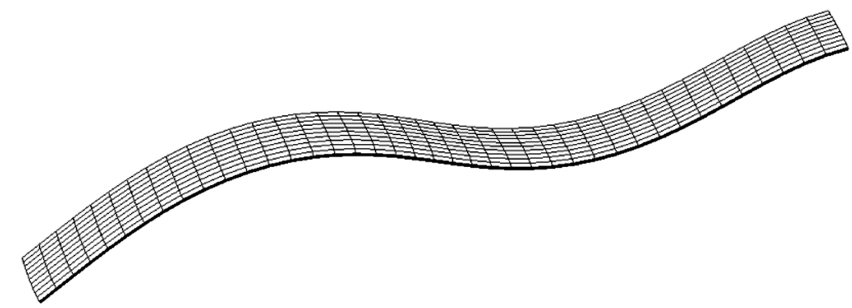

Fig. A4 Fourth mode (third flat bending, $6.20 \mathrm{~Hz}$ ).

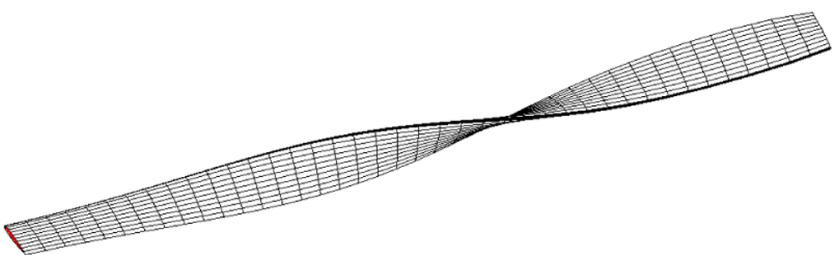

Fig. A5 Fifth mode (second torsion, 6.94 Hz).

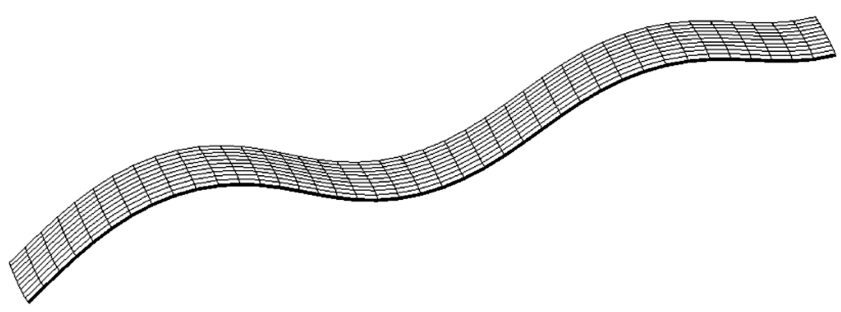

Fig. A6 Sixth mode (fourth flat bending, $12.4 \mathrm{~Hz}$ ).

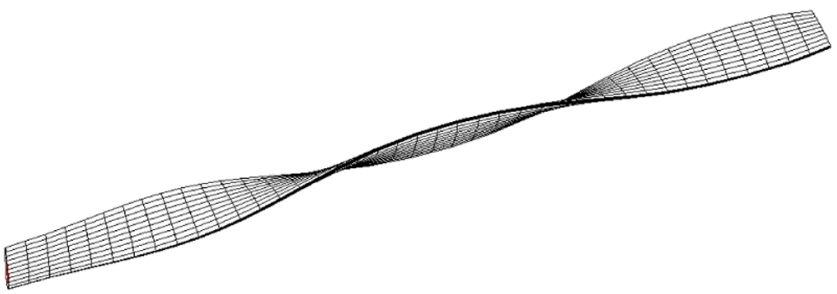

Fig. A7 Seventh mode (third torsion, 17.4 Hz).

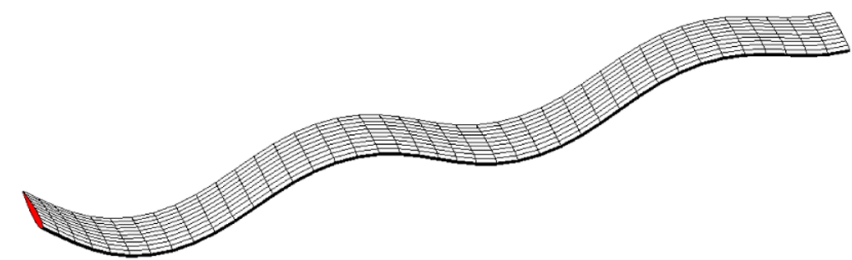

Fig. A8 Eighth mode (fifth flat bending, $20.8 \mathrm{~Hz}$ ).

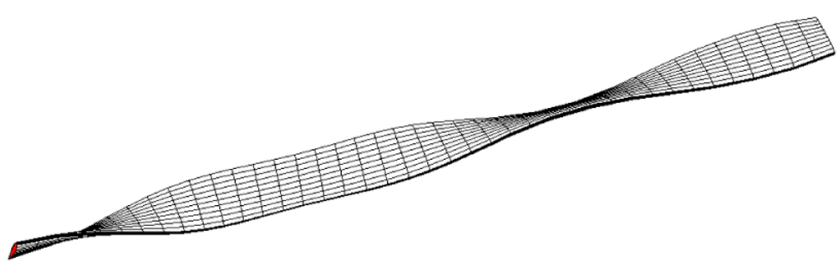

Fig. A9 Ninth mode (fourth torsion, $28.8 \mathrm{~Hz}$ ).

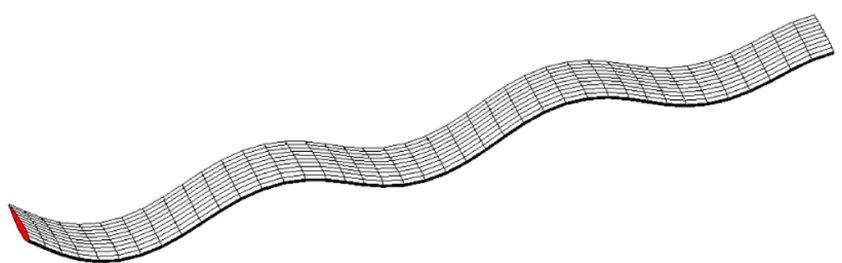

Fig. A10 Tenth mode (sixth flat bending, 31.6 Hz). 


\section{References}

[1] Patil, M. J., Hodges, D. H., and Cesnik, C. E. S., "Nonlinear Aeroelasticity and Flight Dynamics of High-Altitude Long-Endurance Aircraft," Journal of Aircraft, Vol. 38, No. 1, 2001, pp. 88-94. doi: $10.2514 / 2.2738$

[2] Livne, E., and Weisshaar, T. A., "Aeroelasticity of Nonconventional Airplane Configurations: Past and Future," Journal of Aircraft, Vol. 40, No. 6, 2003, pp. 1047-1065. doi: $10.2514 / 2.7217$

[3] Shearer, C. M., and Cesnik, C. E. S., "Nonlinear Flight Dynamics of Very Flexible Aircraft," Journal of Aircraft, Vol. 44, No. 5, 2007, pp. $1528-1545$. doi:10.2514/1.27606

[4] Su, W., and Cesnik, C. E. S., "Nonlinear Aeroelasticity of a Very Flexible Blended-Wing-Body Aircraft," Journal of Aircraft, Vol. 47, No. 5, 2010, pp. 1539-1553. doi:10.2514/1.47317

[5] Su, W., and Cesnik, C. E. S., "Dynamic Response of Highly Flexible Flying Wings," AIAA Journal, Vol. 49, No. 2, 2011, pp. 324-339. doi:10.2514/1.J050496

[6] Bauchau, O. A., and Hong, C. H., "Nonlinear Composite Beam Theory," Journal of Applied Mechanics, Vol. 55, No. 1, 1988, pp. 156-163. doi:10.1115/1.3173622

[7] Hodges, D. H., "A Mixed Variational Formulation Based on Exact Intrinsic Equations for Dynamics of Moving Beams," International Journal of Solids and Structures, Vol. 26, No. 11, 1990, pp. 1253-1273. doi:10.1016/0020-7683(90)90060-9

[8] Palacios, R., and Cesnik, C. E. S., "Geometrically Nonlinear Theory of Composite Beams with Deformable Cross Sections," AIAA Journal, Vol. 46, No. 2, 2008, pp. 439-450. doi: $10.2514 / 1.31620$

[9] Hodges, D. H., "Geometrically Exact, Intrinsic Theory for Dynamics of Curved and Twisted Anisotropic Beams," AIAA Journal, Vol. 41, No. 6, 2003, pp. 1131-1137. doi: $10.2514 / 2.2054$

[10] Zupan, D., and Saje, M., "Finite-Element Formulation of Geometrically Exact Three-Dimensional Beam Theories Based on Interpolation of Strain Measures," Computer Methods in Applied Mechanics and Engineering, Vol. 192, Nos. 49-50, 2003, pp. 5209-5248. doi:10.1016/j.cma.2003.07.008

[11] Gams, M., Planinc, I., and Saje, M., "The Strain-Based Beam Finite Elements in Multibody Dynamics," Journal of Sound and Vibration, Vol. 305, Nos. 1-2, 2007, pp. 194-210. doi:10.1016/j.jsv.2007.03.055

[12] Su, W., and Cesnik, C. E. S., "Strain-Based Geometrically Nonlinear Beam Formulation for Modeling Very Flexible Aircraft," International
Journal of Solids and Structures, Vol. 48, Nos. 16-17, 2011, pp. 2349 2360. doi:10.1016/j.ijsolstr.2011.04.012

[13] Palacios, R., Murua, J., and Cook, R., "Structural and Aerodynamic Models in Nonlinear Flight Dynamics of Very Flexible Aircraft," AIAA Journal, Vol. 48, No. 11, 2010, pp. 2648-2659. doi:10.2514/1.J050513

[14] Tang, D., and Dowell, E. H., "Experimental and Theoretical Study on Aeroelastic Response of High-Aspect-Ratio Wings," AIAA Journal, Vol. 39, No. 8, 2001, pp. 1430-1441. doi: $10.2514 / 2.1484$

[15] Tang, D., and Dowell, E. H., "Experimental and Theoretical Study of Gust Response for High-Aspect-Ratio Wing," AIAA Journal, Vol. 40, No. 3, 2002, pp. 419-429. doi: $10.2514 / 2.1691$

[16] Tang, D., and Dowell, E. H., "Limit-Cycle Hysteresis Response for a High-Aspect-Ratio Wing Model," Journal of Aircraft, Vol. 39, No. 5, 2002, pp. 885-888. doi: $10.2514 / 2.3009$

[17] Patil, M. J., and Althoff, M., "Energy-Consistent, Galerkin Approach for the Nonlinear Dynamics of Beams Using Mixed, Intrinsic Equations," 47th AIAA/ASME/ASCE/AHS/ASC Structures, Structural Dynamics, and Materials Conference, AIAA Paper 2006-1737, 2006.

[18] Palacios, R., "Nonlinear Normal Modes in an Intrinsic Theory of Anisotropic Beams," Journal of Sound and Vibration, Vol. 330, No. 8, 2011, pp. 1772-1792. doi:10.1016/j.jsv.2010.10.023

[19] Cesnik, C. E. S., and Brown, E. L., "Modeling of High Aspect Ratio Active Flexible Wings for Roll Control," 43rd AIAA/ASME/ASCE/AHS/ ASC Structures, Structural Dynamics, and Materials Conference, AIAA Paper 2002-1719, 2002.

[20] Cesnik, C. E. S., and Su, W., "Nonlinear Aeroelastic Modeling and Analysis of Fully Flexible Aircraft," 46th AIAA/ASME/ASCE/AHS/ASC Structures, Structural Dynamics, and Materials Conference, AIAA Paper 2005-2169, 2005.

[21] Palacios, R., and Cesnik, C. E. S., "Cross-Sectional Analysis of Nonhomogeneous Anisotropic Active Slender Structures," AIAA Journal, Vol. 43, No. 12, 2005, pp. 2624-2638. doi:10.2514/1.12451

[22] Peters, D. A., and Johnson, M. J., "Finite-State Airloads for Deformable Airfoils on Fixed and Rotating Wings," Proceedings of Symposium on Aeroelasticity and Fluid Structure Interaction Problems, ASME Winter Annual Meeting, Vol. 44, edited by Friedmann, P. P., and Chang, J. C. I., American Society of Mechanical Engineers, New York, 1994, pp. 1-28.

[23] MSC.Nastran 2004 Quick Reference Guide, Vol. 1, MSC. Software Corp., Santa Ana, CA, 2003. 